PFC/JA-86-62

\title{
Diagnosis of Mildly Relativistic Electron Velocity Distributions By Electron Cyclotron Emission in the Alcator C Tokamak
}

\author{
I. H. Hutchinson and K. Kato \\ Plasma Fusion Center \\ Massachusetts Institute of Technology \\ Cambridge, MA 02139
}

December 1986

Submitted to: American Institute of Physics

This work was supported by the U. S. Department of Energy Contract No. DE-AC0278ET51013. Reproduction, translation, publication, use and disposal, in whole or in part by or for the United States government is permitted.

By acceptance of this article, the publisher and/or recipient acknowledges the U. S. Government's right to retain a non-exclusive, royalty-free license in and to any copyright covering this paper. 


\title{
DIAGNOSIS OF MILDLY RELATIVISTIC ELECTRON VELOCITY DISTRIBUTIONS BY ELECTRON CYCLOTRON EMISSION IN THE ALCATOR C TOKAMAK
}

\author{
I.H. Hutchinson and K. Kato \\ Plasma Fusion Center and Department of Nuclear Engineering \\ Massachusetts Institute of Technology \\ Cambridge, Massachusetts, 02139 U.S.A.
}

\begin{abstract}
Electron cyclotron emission from nonthermal electron distributions has been measured with a vertical view and control of multiple reflections. The observed intensities at the first few harmonics provide information which can be used to deduce the electron distribution function. Although harmonic overlap restricts the detail which can be obtained when the distribution is very energetic, a fitting procedure enables useful results to be obtained. The distribution functions during ohmic runaway and lower hybrid current drive and heating are presented. The current drive distributions are not inconsistent with theoretical Fokker-Planck calculations, giving perpendicular and parallel 'temperatures' around $60 \mathrm{keV}$ and $200 \mathrm{keV}$ respectively. The runaway distributions are quite similar.
\end{abstract}




\section{INTRODUCTION}

In plasmas with anisotropic velocity distribution of electrons, the knowledge of the exact shape of the distribution is of great interest. In lower hybrid heated tokamak plasmas for example, the electron 'tail' which receives almost all the radio frequency (RF) energy may contain a considerable fraction of the total plasma kinetic energy, as well as carrying essentially all the current. Hence, diagnostics to measure $f(\vec{p})$ in these situations are essential to the understanding of the plasma behavior.

Since the ECE spectrum is very sensitive to high energy electron populations, it is well suited for their detection; however detailed analysis of the distribution function has proven very difficult.

Theoretical expressions exist for the spontaneous electron cyclotron emissivity from arbitrary energetic distributions, taking into account the effect of the cold background plasma [1]. Numerical calculations provide insight into the variation in the emissivity with the propagation angle, plasma density, and other parameters of the model distribution, e.g., the Maxwellian temperature and its shift [2]. Theoretical works narrower in focus have investigated anisotropic distributions of specific types (e.g., runaway and RF current drive) to provide approximate analytical relationships between the emission and the distribution $[3,4]$. These allow simplified interpretations which are, in some cases, made possible by the a priori assumption of the distribution shape based on the physical process of its creation (e.g., the lower hybrid current drive wave couples to electrons within a specific energy range). It is difficult to apply these numerical and analytical models to the actual measurements because the relationship between the observed intensity and the local emissivity depends on the specifics of the experimental configuration, including for example multiple reflections of the radiation. Although numerical investigations 
taking into account the tokamak configuration exist $[5,6]$, such considerations are probably best treated on a case-by-case basis.

Broadly speaking, the nonthermal emission spectrum can be divided into two main regions. At low harmonics, discrete harmonic structure in the spectrum remains, while at high harmonics the spectrum becomes essentially a continuum owing to extreme harmonic overlap. The continuum spectral region is less sensitive to the exact viewing geometry in general, but it provides only two spectral slopes (extraordinary $(X)$ and ordinary $(O)$ mode). Therefore, the information about $f(\vec{p})$ that can be deduced is limited. The discrete harmonic measurement has the advantage of a possibility for providing more extensive information but this depends critically on controlling the field of view.

Continuum spectra have been used to measure a 'temperature' and a loss-cone or an anti-loss-cone angle on EBT[7] and PLT[8]. Theoretical prescriptions for distribution function determination from the continuum have been given. These include the identification of parameter (temperature and characteristic pitch-angle) space for which either the polarization ratio or the harmonic slope is a sensitive measurement of the parameters, supported by tabulation of numerical results[9,10,11].

The use of low harmonic, discrete spectra to obtain the distribution has been tried in both mirrors and tokamaks. In mirrors with $\mathrm{MeV}$ electrons $[12,13], \beta=$ $\beta_{\perp}$ is assumed and a profile of $f(\gamma)$ is deduced from the measurement of the low harmonic spectrum. In toroidal devices, nonthermal spectra observed using a radial view have been obtained from numerous machines e.g. $[14,15]$. The interpretation is notoriously difficult however, since nonthermal emission from the center and thermal reabsorption from the outboard side of the toroidal plasma compete at the same frequencies. In PLT, a vertical view was used more recently to measure transmission and emission at the down-shifted first harmonic frequency [17]. These measurements avoided many of the difficulties inherent in the radial view but the results were fitted by a somewhat artificially constrained distribution function form. 
Multiple wall reflections contaminate most measurements [18]. For the continuum measurement, the use of the polarization ratio $I^{+} / I^{-}$(where $I^{+}$and $I^{-}$ are the ordinary and extraordinary mode intensities respectively) requires careful avoidance of polarization cross contamination, which is only rarely achieved. The uncertainty of the propagation angle (in multiply reflected radiation) also obscures the low harmonic structure, despite the numerical models that attempt to account for wall reflections [19].

Attempts to control the wall reflection take on many forms. With two sufficiently large windows at opposite ends of the confinement chamber, a carefully aimed and focussed viewing chord will avoid the wall reflection but some dielectric reflections from the windows will remain [20]. A similar approach is possible with a single aperture and a retro-reflector (usually a spherical mirror) at the target area of the vacuum vessel $[7,21]$.

The Alcator $\mathrm{C}$ vertical viewing ECE diagnostic was conceived in an attempt to obtain more detailed information about the electron velocity distribution from the first few ECE harmonics. A detailed description of the theoretical principles have been given elsewhere [22]. Here, a simplified summary is given to illuminate the concept. When a perpendicular ECE spectrum from a region of constant magnetic field is measured, the frequency broadening is dominated by downward shifts in frequency due to the relativistic mass increase,

$$
\boldsymbol{\omega}=\frac{l \Omega}{\gamma}
$$

where $\Omega=q B / m_{e}$, the rest-mass fundamental cyclotron frequency, and $\gamma=(1-$ $\left.\beta^{2}\right)^{1 / 2}$, the relativistic factor. In this way, electrons can be discriminated according to their total energy. To determine the pitch-angle distribution of the electrons at each of these energies, harmonic or polarization ratios of emissivity, which are 
sensitive to the distribution anisotropy, are measured at each energy level. For example

$$
\frac{j_{3}^{-}(3 \omega)}{j_{2}^{-}(2 \omega)} \text { or } \frac{j_{2}^{+}(2 \omega)}{j_{2}^{-}(2 \omega)}
$$

are measured. Here, $j_{l}^{ \pm}(\omega)$ is the emissivity from the $l$-th harmonic in the ordinary $(+)$ or extraordinary $(-)$ mode at frequency $\omega$. With this anisotropy information in hand, a single harmonic spectrum can then be used to deduce the absolute number density of the distribution for each energy. Perpendicular emission cannot provide information on any parallel-anti-parallel asymmetry, but measures the 'forward/backward-average' of the distribution. This is an important limitation, but does not prevent us from obtaining information which is still very useful.

We use a parameterization of the distribution function which lends itself to the analysis we shall use. We write

$$
f(\vec{p})=f_{p}(p) f_{\theta}\left(p, \theta_{p}\right)
$$

where $f_{p}$ is the phase space density of electrons at momentum $p$ and $f_{\theta}$ is the pitch-angle $\left(\theta_{p}\right)$ distribution of electrons at $p$, for which we assign a form

$$
f_{\theta}=L \exp \left\{-\Lambda \cos ^{2} \theta_{p}\right\}
$$

where $\mathrm{L}$ is the normalization constant such that

$$
\int_{0}^{\pi} f_{\theta}\left(p, \theta_{p}\right) \sin \theta_{p} d \theta_{p}=2
$$

A single anisotropy factor, $\Lambda(p)$, thus determines the pitch angle variation of the distribution at each p. A positive $\Lambda$ implies a loss-cone type of distribution (oblate in shape), and a negative $\Lambda$ implies an enhancement in the parallel direction (prolate). 
When $\Lambda=0$, the pitch-angle distribution is isotropic. In the case of extreme anisotropies, $|\Lambda| \gg>1$, this choice of $f_{\theta}$ approximates a guassian spread along $p_{\|}=$const. $(\Lambda<<-1)$, or $p_{\perp}=$ const. $(\Lambda>>1)$.

The critical requirement for this diagnostic is that frequency broadening mechanisms other than the relativistic broadening be eliminated or contained to manageable levels. It is for this reason that the vertical view through the center of a tokamak plasma is employed to suppress field and Doppler broadening.

To insure isolation of this viewed region, an efficient microwave absorber (viewing dump) [23] is used to suppress the wall reflected radiation. Such a dump must be placed inside the Alcator $\mathrm{C}$ vacuum chamber, which requires it to be compact, vacuum compatible, and to be able to withstand the high particle and heat fluxes. The restricted access of Alcator $\mathrm{C}$ also necessitated novel approaches to the focussing optics design, as described elsewhere [24].

In Section II, the apparatus and some representative measurements obtained from it are presented to show the performance of the diagnostic. In Section III, the fairly complicated methods of distribution function determination, necessitated by the presence of harmonic superpositions, are discussed. Results of $f(\vec{p})$ analyses are presented in Section IV for representative lower hybrid current drive and low density ohmic discharges.

\section{MEASUREMENT}

The vertically viewed ECE is measured by the apparatus whose elevated and plan views are shown in Fig. 1. This system was designed with an emphasis on achieving a narrow collimated view through the center of the plasma[24]. Compact, vacuum compatible viewing dumps have been developed [23] and placed at the bottom of the vacuum chamber. The frequency spectrum is obtained every $15 \sim 20$ 
ms by the rapid scan polarizing Michelson interferometer - InSb detector system, calibrated to an accuracy of approximately $\pm 15 \%$ in the range $200 \sim 700 \mathrm{GHz}$ ( $\Omega \sim 3 \Omega$ for typical Alcator Operation).

Maximum use of the limiter shadow region of the Alcator vacuum chamber was made at each stage of operation so that the dump size differed significantly between lower hybrid current drive experiments at $a$ (minor radius) $=16.5 \mathrm{~cm}$ for which the dump dimensions were $7 \times 7 \mathrm{~cm}$ and low density ohmic discharge experiments at $a=11.5 \mathrm{~cm}$ for which the dump dimensions were $14 \times 14 \mathrm{~cm}$. Comparison with and without the dump is available only for the $a=16.5 \mathrm{~cm}$ plasma, while the polarization ratio measurements are available only with the $a=11.5 \mathrm{~cm}$ plasma.

In Fig. 2 we show two representative extraordinary mode ECE spectra covering the first three harmonics from $B_{T}=8 \mathrm{~T}$ thermal plasmas with the same discharge conditions having $a=16.5 \mathrm{~cm}$. Trace A (dotted line) was taken with a stainless steel flat plate in place of the viewing dump and Trace B (solid line) was taken with the Macor viewing dump. The cyclotron frequency at the center of the plasma is $220 \mathrm{GHz}(\Omega)$.

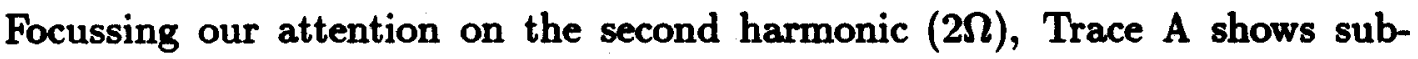
stantial emission from essentially all major radii occupied by the plasma ( $48 \leq$ $\mathrm{R}(\mathrm{cm}) \leq 80,350 \leq \nu(\mathrm{GHz}) \leq 580)$. In this respect, the emission is similar to that obtained by the horizontal configuration; multiple reflections and the high reflectivity of the stainless steel are responsible for extending the view. The depression in the spectrum in the range $430 \sim 460 \mathrm{GHz}$ is caused by the optically thick resonance effect where the resonance layer inside the viewing chord decreases the optical penetration [25]. Spectra similar to this have been observed previously in other vertical viewing experiments [26].

The second harmonic emission of Trace B is dramatically different from A, and now most of the intensity is concentrated in a narrow peak at $\mathbf{4 4 0} \mathbf{G H z}$ with 
a full-width-at-half-maximum (FWHM) of $24 \mathrm{GHz}$. The suppression of emission outside this peak attests to the effectiveness of the dump in absorbing multiply reflected emissions that land on it, preventing reflections into the viewing chord. The FWHM corresponds to $\approx 3.5 \mathrm{~cm}$ in the major radius direction and we attribute the broadening entirely to the magnetic field gradient. This width (presumably near the center of the plasma in the vertical direction) is consistent with diffraction calculations [24] for our antenna pattern.

The finite intensity outside the narrow peak in Trace B is attributed to reflections from the dump, or more likely its frame, since we found that the antenna pattern is slightly larger than dump size of $7 \times 7 \mathrm{~cm}$. The effectiveness of the system in removing radiation from outside the viewing chord is defined as

$$
\eta_{\text {removal }}=\frac{I_{A}(\omega)-I_{B}(\omega)}{I_{A}(\omega)}
$$

where subscripts $A$ and $B$ refer to the traces, and the range of $\omega$ excludes the resonance inside the viewing chord. Measurements of $\eta_{\text {removal }}$ from the spectra of Fig. 2 and other traces in the second harmonic frequency range show $\eta_{\text {removal }}=$ $90 \pm 5 \%$. The magnitude of the variation of the effectiveness over the frequency range of interest $(200 \sim 700 \mathrm{GHz})$ is believed to be within the uncertainty.

The third harmonic profile taken without the viewing dump does not show the depression at the frequency corresponding to the plasma center. This result can be explained by assuming $\tau_{3}<<1$, where $\tau_{3}$ is the optical depth at the third harmonic, just as in the radial view. Widths of the third harmonic profiles with and without the dump are not very different, probably because the emission in this harmonic is already heavily weighted towards the region inside the viewing chord for two reasons; (1) the stainless steel plate tends to act as a more efficient retro-reflector at these wavelengths, and (2) since $j_{3} \propto n T^{3}$ for optically thin emission. Thus, it 
is difficult to calculate accurately the system effectiveness in the manner employed for the second harmonic emission.

The interpretation of the first harmonic spectra is difficult due to the presence of various layers, such as the upper-hybrid and the right-hand cut-off layers. We shall therefore note only that emission from the first harmonic exists at frequencies corresponding to the resonance in the entire plasma $(175 \sim 290 \mathrm{GHz})$ for both measurements, although with an intensity not too different from the noise level. The emission measured without the dump is larger by a factor of two probably because the dump contributes to reduction of the effective wall reflectivity regardless of whether the viewing chord sees it directly or not.

In Fig. 3(a), we plot a nonthermal spectrum during lower hybrid heating measured without the viewing dump. In this spectrum, down-shifted first harmonic emission is seen below $220 \mathrm{GHz}(\Omega)$, and the broad depression at $\Omega$ is due to the thermal absorption. Above $250 \mathrm{GHz}$, the emission shows a sharp rise with frequency as the thermal resonance shifts out of the plasma. The emission at these frequencies and up to $440 \mathrm{GHz}(2 \Omega)$ is primarily the down-shifted second harmonic emission, but at $360 \mathrm{GHz}$, the intensity starts to decrease sharply, reaching a minimum at

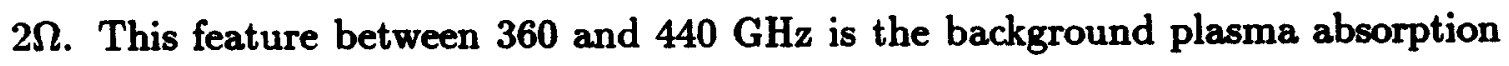
profile where the multiply reflected radiation intensity is reduced by the thermal reabsorption layer present in the plasma. We note that at the outboard edge of the plasma, the second harmonic cyclotron frequency is $350 \mathrm{GHz}$, so that the onset of thermal plasma reabsorption agrees well with the plasma edge frequency. That the intensity above $2 \Omega$ does not recover to the level seen below $2 \Omega$ in a symmetric manner probably indicates that the Doppler broadening, normally symmetric in frequency upshift and downshift, is not a strong contributor, i.e., $\Delta \theta / \theta<<1$. Thus, we believe the emission above $2 \Omega$ to be mostly from the down-shifted third harmonic. The dip at $560 \mathrm{GHz}$ is due to an atmospheric water vapor absorption 
line, whose effect is to block out the frequency spectrum, limiting the distribution function energy range over which the spectrum can be interpreted quantitatively with full confidence.

In Fig. 3(b), we show a nonthermal spectrum of the extraordinary mode during lower hybrid heating measured with the $7 \times 7 \mathrm{~cm}$ dump in place. The down-shifted first harmonic is seen as before, although the depression at $\Omega$ is not as wide as in (a), possibly partly because of the lower density. The narrow peak at $270 \mathrm{GHz}$ is we interpret as due to the right-hand-cut-off $\left(\omega_{R}\right)$ close to the line-of-sight reflecting the antenna pattern away from the dump and hence enhancing the observed intensity. The dip at $380 \mathrm{GHz}$ is due to a weak water vapor absorption line, while the stronger absorption at $560 \mathrm{GHz}$ is prominent in this spectrum as well. Ignoring the $380 \mathrm{GHz}$ dip, the emission increases with frequency from just above the $\omega_{R}$ peak all the way up to $420 \mathrm{GHz}$, indicating that no substantial thermal plasma reabsorption is present. A dramatic discontinuity is seen at $2 \Omega$, and the substantially lower emission on the high frequency side is consistent with expectations of a purely downshifted emission. The finite intensity of the down-shifted third harmonic emission continuing from above $2 \Omega$ to below is an indication that accounting of harmonic superpositions is required in the $f(\vec{p})$ analysis.

For the $a=11.5 \mathrm{~cm}$ plasma, measurements of polarization ratios from thermal plasmas indicate a higher ordinary mode intensity $\left(\mathrm{I}^{+} / \mathrm{I}^{-}=0.05\right)$ in the third harmonic that can be explained by the theoretical emissivity $[25,27]$; although much less than is observed without the dump $\left(I^{+} / I^{-} \sim 0.5\right)$. The $5 \%$ polarization ratio is attributed primarily to slight depolarization in the optical system. When the ordinary mode nonthermal spectra are measured, their intensity is corrected according to the prescription,

$$
I^{+\prime}(\omega)=I^{+}(\omega)-0.05 I^{-}(\omega)
$$


to account for the depolarization. As justification for this process, we note that in the thermal plasmas the depolarization is observed to be approximately independent of frequency, and whether it happens along the optical train or at the dump, the source spectrum (of depolarization) is predominantly the extraordinary mode emission.

In summary, the apparatus is successful in limiting the radiation from outside the viewing chord to $\leq 10 \%$, while its limit on the depolarization is $5 \%$, based on observations of thermal plasma emissions. The ordinary mode nonthermal emission is thus corrected accordingly, while the extraordinary mode emission is left as is since no concrete signs of multiple reflections can be observed.

\section{METHOD OF DIAGNOSIS}

In principle, obtaining the values of harmonic or polarization ratios provides easy access to the $f(\vec{p})$ description. In practice however, harmonic superpositions, which occur when the $l$-th harmonic downshifts below the $(l-1)$-th harmonic at $\gamma=l /(l-1)$, destroy the unique correspondence between the frequency and the energy. For the second and the third harmonics that we are interested in, this occurs at $511 \mathrm{keV}$ and $256 \mathrm{keV}$, respectively.

In lower hybrid heating plasmas at high densities such as that of Fig. 3(b), the harmonic superposition is small and $f_{p}$ and $\Lambda$ at each energy can be found without overall constraints on their form. The results of such an analysis are reported elsewhere [28]. In many cases however, the harmonic superposition is so severe that such a method breaks down and an aggregate analysis must be performed on the spectrum, from which several parameters that specify the distributions are found. We shall concentrate here on these cases.

Our approach basically is to produce a reasonable description of $f(\vec{p})$ still based on the $f_{p}$ and $\Lambda$ form, and use a few parameters which can reasonably approximate 
the measurement. To this end, examinations of several candidate forms for $f_{p}$ and $\Lambda$ led to the selection of

$$
f_{p}=f_{o} \exp \left\{-\frac{E}{T}+\frac{C}{E}\right\}
$$

and

$$
\Lambda=A p^{B}
$$

where the variables $f_{o}, \mathrm{~T}, \mathrm{C}, \mathrm{A}$, and B are to be 'measured' [29]. In Eqn.8, the $C$-parameter, whose inclusion is essential to improving the fit, simulates the low energy transition between the thermal bulk and the energetic tail. The form of $\Lambda$ is a simple power of $p$, providing smoothness and flexibility.

The approach used is to try to optimize the five parameters such that the electron cyclotron emission spectrum computed from the model distribution best fits the measured spectrum over specified ranges of frequencies. In consideration of the highly energetic nature of the distributions, emission from $l=2$ to 14 harmonics are summed in the calculation, while the range of fitted frequency is usually within $1 \leq \omega / \Omega \leq 3$. Since the nonthermal emission measured occurred during low densities, typically $\omega_{\text {pe }}^{2} / \Omega^{2} \leq 0.05$, the tenuous plasma approximation was used for the emissivity calculations.

Two computer codes are used: BESTFIT and BESTPAIR. The former fits to the harmonic ratio of a single mode spectrum while the latter fits to the polarization ratio of ordinary and extraordinary modes. They use look-up tables of normalized $j$, the emissivity, vs. $\Lambda$ to execute the optimization.

The optimization criterion is the $\chi_{\nu}^{2}$ parameter[30], where

$$
\chi_{\nu}^{2}=\frac{1}{\nu} \sum_{i=1}^{N}\left\{\frac{\left(I_{e}\left(\omega_{i}\right)-I_{c}\left(\omega_{i}\right)\right)^{2}}{\sigma^{2}\left(I_{e}\left(\omega_{i}\right)\right)}\right\}
$$


where $\nu=N-m$ and where $\mathrm{N}$ is the total number of fitted points, which we take to be the frequency range of the fit divided by the frequency resolution $(\sim 20 \mathrm{GHz})$, and $m$ is the number of independent variables (five in our case). $I_{e}$ and $I_{c}$ are the experimental and computed intensities at $\omega_{i}$, and $\sigma^{2}\left(I_{e}\left(\omega_{i}\right)\right)$ is the square of the unnormalized uncertainty at $\omega_{i}$ (typically $\pm 15 \%$ ). A value of $\chi_{\nu}^{2}$, of approximately unity implies a statistically sound fit.

These codes were checked for absolute consistency using spectra generated from Tamor's data [2]. A self-consistency check, i.e., the code's ability to recover an arbitrary distribution whose ECE was generated using its own data-base has also been performed, with similar success.

In these diagnoses, the shape of $f(\vec{p})$ is constrained by Eqns. 8 and 9 so that the result is insensitive to narrow frequency fluctuations on the measured spectrum. Instead, the dominant source of uncertainty is the relative sensitivity calibration of the down-shifted second harmonic range of frequencies, $\Omega \leq \omega \leq 2 \Omega$, to the downshifted third harmonic range of frequencies, $2 \Omega \leq \omega \leq 3 \Omega$, which we believe to be accurate to within $\pm 15 \%$. Thus, in order to investigate this effect, BESTFIT and BESTPAIR were applied to 'distorted' spectra, in which the intensity at the extraordinary mode second harmonic range of frequencies was decreased or increased by $15 \%$. The results of these distorted analyses show up as error limits flanking the true solutions of $f_{p}$ and $\Lambda$. The choice of the extraordinary mode second harmonic as the range of distortion will provide the worst case result since it is the most dominant in intensity, and its magnitude is more directly reflective of the single harmonic emissivity due to the smaller superposition compared to the higher harmonics. 


\section{RESULTS}

\section{A. Lower Hybrid Current Drive}

In lower hybrid current drive experiments on Alcator $\mathrm{C}$, using as much as $1 \mathrm{MW}$ of $4.6 \mathrm{GHz}$ lower hybrid waves injected by phased waveguide arrays, non-inductive current of up to $I_{p} \simeq 200 \mathrm{kA}$ was driven. (Heating of $\Delta T_{e} \sim \Delta T_{i} \sim 1 \mathrm{keV}$ has also been achieved) $[31,32]$.

Fig. 4 shows the discharge from which the extraordinary mode vertical ECE spectrum is analyzed. Five traces show the vertical ECE raw interferogram [37] data, plasma current $\left(I_{p}\right)$, line-averaged density $\left(\bar{n}_{e}\right)$, hard X-ray, and the loop voltage $\left(V_{\text {loop }}\right)$. The radio frequency pulse is indicated on the $I_{p}$ trace, while the specific interferogram scan to be analyzed is indicated by an arrow in the vertical ECE trace.

The consequences of the RF pulse are apparent in all but the $\bar{n}_{e}$ trace. The vertical ECE signal undergoes an order of magnitude increase, while a similar phenomenon is observed in the hard X-ray signal. The current sustainment by the RF wave is evidenced by the flattening of $I_{p}$ profile and decrease in $V_{\text {loop }}$ (to zero) during the RF pulse. The nonthermal vertical ECE signal reaches steady-state within one scan time $(<15 \mathrm{~ms})$ in this discharge. Even though current sustainment by the RF generated tail implies a high energy electron distribution with strong asymmetry in the $p_{\|}$direction, this asymmetry cannot be measured by our configuration as discussed earlier, so that the measured result should be interpreted as the average of the forward and the backward distributions.

In Fig. 5, we show two vertical ECE spectra, just before and during the RF. A noteworthy feature of this figure is the vast difference in intensity of the two traces. Clearly, nonthermal emission from the tail electrons dominate in the upper trace. The shape of the nonthermal ECE is also quite different from the lower hybrid heating discharge spectrum of Fig. 3(b), where even though there is depression at the 
second harmonic rest mass frequency, the intensities above and below this frequency do not exhibit the sharp 'step' feature, seen with the heating discharge spectrum of Fig. 3(b). This is a consequence of the much broader relativistic spread, resulting in strong harmonic superpositions. The depression at $2 \Omega$ is created by both the addition of down-shifted second harmonic emission to the third just below $2 \Omega$, and the thermal reabsorption at $2 \Omega$. The peak above $\Omega$ shows the $\omega_{R}$ effect discussed previously, and below $\Omega$ is the down-shifted first harmonic emission similar to the higher harmonics. The water vapor absorption line at $560 \mathrm{GHz}$ is interpolated linearly. This procedure gives a good estimate of the intensity to within the calibration uncertainty. The spectrum above $3 \Omega$ is noise dominated because of the low response of the detection system.

Fig. 6 shows the measured lower hybrid current drive spectrum and the computer generated ECE. The frequency range over which the fit was optimized according to the above prescription is shown by the two horizontal lines near the abscissa. They correspond to $1.2 \leq \omega / \Omega \leq 1.9$ and $2.2 \leq \omega / \Omega \leq 2.7$, covering much of the second and the third harmonic down-shifted emission. The substantial harmonic overlap, especially for the third harmonic, is evident.

The $f_{p}$ and $\Lambda$ profiles in the range $10 \leq \mathrm{E}(\mathrm{keV}) \leq 250$ that generate this spectrum are shown in Fig. 7. Energy is plotted on the abscissa in these plots so that Maxwellian temperature can be obtained by straightforward slope fitting in the $f_{p}$ plot: No ambiguity arises because of the one-to-one correspondence between the energy and momentum,

$$
\left.E=m_{e} c^{2}\left[p^{2}+1\right)^{1 / 2}-1\right]
$$

(We measure $\mathrm{p}$ in natural units of $m_{e} c$ ).

These results are of a fit with $T=100 \mathrm{keV}, C=40 \mathrm{keV}, A=-2.2$, and $B=2.0$. At the low energy end, the plot of $f_{p}$ shows a gradual transition in 
slope from the thermal to the tail, introduced by the finite $C$-parameter. The $\Lambda$ profile shows a somewhat artificially constrained form, with $-3<\Lambda<0$, indicating significant parallel enhancement consistent with expectations of the existence of a current carrying tail. The dashed lines flanking the solid traces in Fig. 7 show the possible regions of $f_{p}$ and $\Lambda$ values, obtained from an aggregate error analysis discussed above. The contour plot of this distribution is given in Fig. 8.

\section{B. Low Density Ohmic Discharge}

Vertical ECE data from low density ohmic discharges were obtained in the $a=11.5 \mathrm{~cm}$ plasma, with the larger viewing dump. Pairs of identical discharges have been used to obtain data from the ordinary and the extraordinary modes. Thus, for this discharge, analysis of two nonthermal spectra obtained from a set of identical discharges is carried out using both the three-to-two harmonic ratio of the extraordinary mode and the polarization ratio at the second harmonic. As noted previously, the ordinary mode spectrum used is processed using the prescription of Eq. 7. The ordinary mode harmonic ratio is not used in the analysis because we believe that substantial distortion of this quantity may have resulted from the post-processing.

The discharge traces from which the spectrum is taken is shown in Fig. 9, in a format similar to the previous one. The difference here is that the two detector traces, corresponding to the ordinary and the extraordinary mode measurements from identical discharges, have been included and the loop voltage trace has been removed because of its constancy. The density is extremely low for an Alcator ohmic discharge at $\tilde{n}_{e}<0.3 \times 10^{20} \mathrm{~m}^{-3}$. It is this low density relative to the ohmic induction that promotes part of the electron population to accelerate, causing 'slideaway' [34] or 'runaway' [35] discharges. The vertical ECE signals exhibit strong enhance-

ments due to the nonthermal emission throughout most of the discharge. The onset 
of the nonthermal activity coincides with the hard X-ray signal increase, and other parameters, $I_{p}$, and $\bar{n}_{e}$ both remain roughly constant during the nonthermal activity, indicating 'steady' energetic tail sustainment. Although the hard X-ray trace shows steadily increasing periodic structure, the effect responsible for this appears not to affect the vertical ECE signal, probably because the source of this hard X-ray activity lies outside the line-of-sight, or the electrons causing this effect are of such low density or of high energy that this activity cannot be detected by the ECE system. Essentially identical spectra are obtained during the period $150 \sim 300 \mathrm{~ms}$.

The two vertical ECE spectra are shown in Fig. 10 Fig. 10(b) shows the X-mode which exhibits structures similar to the current drive case of Fig. 5, indicating the presence of emissions from highly relativistic electrons and severe harmonic superpositions. A notable difference is the apparent absence of the $\omega_{R}$ peak, presumably due to the extremely low density of the discharge. The O-mode spectrum shown in Fig. 10(a) has a substantially lower intensity than the X-mode throughout the frequency range, although the harmonic features are similarly identifiable. The $\mathbf{5 6 0}$ $\mathrm{GHz}$ water vapor absorption line dips will be filled in as before, but the ones at 760 $\mathrm{GHz}$ will be left untouched since they are outside of the fitting range.

For the distribution function deduction, we apply the analysis to the harmonic ratio and the polarization ratio individually. Fig. 11 again shows the two spectra, this time plotted on the same scale. The smooth dotted curve overlayed on the measured X-mode spectrum shows the harmonic ratio fit to the $\mathrm{X}$-mode, while the dashed curves in the down-shifted second harmonic range of frequencies show the fit according to the second harmonic polarization ratio. The frequency ranges over which the fittings were performed are indicated by solid lines near the abscissa. These ranges are the same as for the current drive case.

The four distribution parameters (excluding $f_{0}$ ) that produce these fits are: For the harmonic ratio, 


$$
T=100, C=40, A=-1.25, B=1.50 \text {. }
$$

For the polarization ratio,

$$
T=85, C=60, A=-5.0, B=-0.15
$$

As can be seen by the similarity of the fitted curves at the down-shifted second harmonic range of frequencies in the X-mode, parameters that characterize $f_{p}$ (which is sensitive to the fit of a single harmonic profile) are not grossly different. The large differences in $A$ and $B$ arise because $\Lambda$ is sensitive to the ratios rather than the individual harmonic shape.

A single $f(\vec{p})$ that produces a fully acceptable fit with both $I_{3}^{-} / I_{2}^{-}$and $I_{2}^{+} / I_{2}^{-}$ could not be found with the present prescription. For these spectra, and for other low density ohmic discharges in which polarization ratios were measured in general, the result of BESTFIT underestimates the polarization ratio, i.e., the computed O-mode using the BESTFIT- derived $f(\vec{p})$ is weaker than the measured value. The result of BESTPAIR, on the other hand, underestimates the harmonic ratios of both polarizations, i.e., the computed third harmonics using the BESTPAIR derived $f(\vec{p})$ are weaker than the measured values.

The discrepancy between the two distributions is evident in Fig. 12, where both distribution function parameters, along with the aggregate error analysis results, are plotted. In this figure, solid lines show the fits from BESTFIT and BESTPAIR, with labels ' $\mathrm{H}$ ' and ' $\mathrm{P}$ ' referring to the harmonic and the polarization result, respectively. The dotted-line error curves belong to the harmonic ratio result, and the dashed-line error curves belong to the polarization ratio result. The upper error curve for the harmonic ratio in the $\Lambda$ plot is indistinguishable from the $\Lambda=0$ axis. The harmonic ratio error curves are produced using the $\pm 15 \%$ distortion on the second harmonic 
fitting range as before, while for the polarization ratio, distortions of $\pm 25 \%$ were introduced to the $\mathrm{O}$-mode intensity. This $25 \%$ value takes into account, according to the error propagation principle, the uncertainty of the post-processing prescription which consists of the uncertainties in the $5 \%$ contamination value and the $\mathrm{X}$-mode intensity in addition to the uncertainty in the unprocessed O-mode measurement.

In the figure, the larger distortion value of the polarization ratio result manifests in the wider space between the polarization error curves. The shapes of $f_{p}$ from the two fits are quite similar, but with a major discrepancy in the magnitude despite the fact that computed spectra from the two fits agree well in the X-mode second harmonic range of Fig. 11. This is due to the large difference in the magnitude of the pitch angle integrals for each fit brought about by the different $\Lambda$ values. As for the $\Lambda$ values themselves, the discrepancy is substantial. The $\Lambda$ spectrum from the polarization ratio should probably be interpreted as a constant $\Lambda$ of approximately -5. The large negative values of $\Lambda$ at the low energy end is probably an artifact of the fitting process, since the computed O-mode spectrum does not agree with the measurement near $2 \Omega$ anyway. In any case, both fits indicate parallel enhanced distributions as expected, which indicates the presence of high energy electrons accelerated by the toroidal electric field.

The nature of the discrepancy is consistent with the effects of insufficient accounting of superpositions, as discussed elsewhere[25], so that the true parameters for $f(\vec{p})$ probably lie somewhere in between those in Eqns. 12 and 13. Thus, although the examination of the two ratios 'bracket' the probable range of $f_{p}$ and $\Lambda$, precise information is clearly desired.

That calibration uncertainties can cause the discrepancy is evident by noting how the error boundaries from the two results overlap, essentially throughout the energy range for $f_{p}$ and at high energies for $\Lambda$. Thus, uncertainties in the measurement are probably responsible for a part of the discrepancy, and because of this, 
we shall choose to take, as our best estimate, the distribution of the harmonic ratio error curve that lies between the ' $\mathrm{H}$ ' and the ' $\mathrm{P}$ ' solid line curves. The parameters for this distribution are

$$
T=125, C=40, A=-3.2, B=1.30 \text {. }
$$

In Fig. 13(a), we present the contour plot for this distribution. For comparison, the contour plots of the two individual ratio estimates, whose parameters are given in Eqn. 12 and 13 are plotted in Fig. 13(b) and (c). It is seen that the difference between the two individual ratio results in the latter figure is substantial, while the two contours Fig. 13(a) and (b), which are not too dissimilar, show a typical difference as a result of the $15 \%$ aggregate distortion analysis also carried out for the lower hybrid current case.

\section{DISCUSSION}

The results given above provide a complete parameterization of the distribution function in so far as it can be determined from ECE. However, because the form in which we have chosen to describe $f(\vec{p})$ is different from that used in other experimental and theoretical works we now provide a reexpression of our results in ways that make comparisons easier.

In cases such as ours, when a relativistic discussion is essential, there is no obvious 'natural' division of the distribution in parallel and perpendicular directions. For example, a Maxwellian distribution is no longer a Guassian in momentum and so is not a product of separate functions of $p_{\|}$and $p_{\perp}$. Also, the Landau resonance is

$v_{\|}=$constant which is not $p_{\|}=$constant. In view of these difficulties, unanimity in the choice of distribution parameterization is not to be expected. However, we here choose to present our distributions in the form of a parallel momentum distribution: 


$$
F_{\|}\left(p_{\|}\right) \equiv \int_{0}^{\infty} f 2 \pi p_{\perp} d p_{\perp}
$$

and a perpendicular momentum spread:

$$
\left\langle p_{\perp}^{2}\right\rangle_{p \|}=\int_{0}^{\infty} f p_{\perp}^{2} 2 \pi_{\perp} d p_{\perp} / F_{\|}\left(p_{\|}\right)
$$

evaluated on lines of constant $p_{\|}$.

In Figs. 14 to 16 we show plots of these parameters for the three types of plasma we have studied (including the LH heated cases [28] smoothed according to the fitting prescription of Eqs. (8) and (9)).

To reduce this data to simple numbers representing momentum spreads as effective temperatures we adopt the following definitions. The effective perpendicular temperature, $T_{\perp}$, is that number which best fits the expression

$$
f\left(p_{\perp}, p_{\|}\right)=f_{o} \exp \left\{-\frac{m_{e} c^{2}}{T_{\perp}\left(p_{\|}\right)} \sqrt{1+p_{\|}^{2}+p_{\perp}^{2}}\right\}
$$

along $p_{\|}=$const. Similarly $T_{\|}$is chosen to optimize

$$
F_{\|}\left(p_{\|}\right)=F_{o} \exp \left\{\frac{-m_{e} c^{2}}{T_{\|}} \sqrt{1+p_{\|}^{2}}\right\}
$$

Equation (17) has the merit that for an isotropic relativistic distribution $T_{\perp}$ is equal to the distribution temperature. Unfortunately this is not true for $T_{\|}$defined by Eq. (18), but this definition seems most natural. Relating the $T_{\perp}$ to $\left\langle p_{\|}^{2}\right\rangle$ we recognize that for $T_{\perp} \ll m_{e} c^{2}$ the Maxwellian form can be written approximately as proportional to $\exp \left[-p_{\perp}^{2} m_{e} c^{2} / 2 T_{\perp} \gamma_{\|}\right]$where $\gamma_{\|}=\sqrt{1+p_{\|}^{2}}$. Therefore the approximate relationship between $T_{\perp}$ and $\left\langle p_{\perp}^{2}\right\rangle$ is:

$$
T_{\perp} \simeq \frac{m_{e} c^{2}}{2 \gamma_{\|}}\left\langle p_{\perp}^{2}\right\rangle
$$


Notice the additional factor $\gamma_{\|}$by which this relationship differs from the normal nonrelativistic one.

In Table I we summarize $T_{\|}, T_{\perp}$, the total tail density, $n_{t a i l}\left(\int F_{\|} d p_{\|}\right)$and several discharge parameters for the three different plasmas. The $T_{\perp}$ value is an average over the energy range. The uncertainties are determined from the extreme of $f$ obtained in the fitting process. The +0 attributed to $T_{\|}$in the ohmic case is due to the fact that we have taken the optimum fit to lie between the two solid curves of Fig. 12, both of which have lower $T_{\|}$than the intermediate fit.

In seeking to compare these results with theoretical expectations, particularly for the lower hybrid cases, the uncertainties, both in our results and in the theory of the tail, make decisive conclusions difficult. In all three cases, theory suggests that the tail of the distribution should be very flat in the parallel direction. Our results confirm this trend but tend to show rather greater negative slope on $F_{\|}$than Fokker-Planck calculations indicate[36]. Within the uncertainty of our measurements, however, $F_{\|}$could be flat, for the current drive and runaway cases. It should be recalled, in addition, that what we measure is the chord-averaged distribution function, while all the detailed 2-D Fokker-Planck calculations deal with uniform plasmas. It is quite possible that spatial variation of $F_{\|}$gives rise to a greater slope on the chord averaged measurement than would be observed locally.

Of particular interest is the value of $T_{\perp}$. We observe rather higher values than have been obtained in most Fokker-Planck code calculations for lower hybrid current drive. However, in the codes, it is observed that $T_{\perp}$ is strongly dependent on the assumed wave $n_{\|}$spectral range within the plasma. Since this range may be completely different from the launched spectrum [37] it is difficult to make meaningful quantitative comparisons. However, we may gain an estimate of the highest possible resonant $p_{\|}$(at $p_{\perp}=0$ ) from considerations of wave accessibility. These give $n_{\|} \simeq 1.4 \Rightarrow p_{\|} \simeq 1.0$, and $n_{\|} \simeq 1.25 \Rightarrow p_{\|} \simeq 1.33$ for the heating and current-drive 
cases respectively, at the discharge center. The bulk temperature is approximately $T_{e}=1.2 \mathrm{keV}$ in both cases so the thermal momentum is $p_{t h}=\sqrt{T_{e} / m_{e} c^{2}} \simeq 0.05$ (in units of $\left.m_{e} c\right)$. Therefore we may reasonably estimate $p_{\|} / p_{t h} \simeq 20$ for the heating and $\simeq 27$ for the current-drive upper momentum limit. Since fully relativistic calculations covering our case appear not yet to have been published we compare with the $2-D$ non-relativistic study of Fuchs et al[38] which indicates (for $Z_{\text {eff }}=1$ ) $T_{\perp} \simeq 30 \mathrm{keV}$ and $T_{\perp} \simeq 45 \mathrm{keV}$ for the two cases in which our measurements give 30 $\mathrm{keV}$ and $60 \mathrm{keV}$. Since the theoretical temperature may be considerably increased by a value of $Z_{\text {eff }}$ greater than 1, we may regard these results as not inconsistent. (Up to $Z_{\text {eff }}=2$ may occur in our experiment).

We conclude, then, that it is probably possible to explain the observed $T_{\perp}$ on the basis of Fokker-Planck modeling without recourse to additional pitch-angle scattering mechanisms in the Lower Hybrid cases; although more detailed theoretical comparisons would be helpful. On the other hand, the ohmic plasmas have distributions strikingly similar to the current drive and in their case the evidence is very strong that the anomalous cyclotron ('anomalous Doppler') instability plays a vital role [39].

Independent measurements of the distribution during lower-hybrid current drive on PLT using $x$-ray Bremsstrahlung [40] have given comparable values of $T_{\perp} / T_{e}$ (though both temperatures were higher by a factor of about 2 ). There it was also concluded that collisional processes could explain the result. A more direct experimental comparison is possible with the x-ray results of Texter [41] on similar Alcator C plasmas. He found $T_{\perp}=85 \mathrm{keV}, T_{\|}=280 \mathrm{keV}$ (in our definitions) as compared with our values of 60 and $200 \mathrm{keV}$. The differences here are probably within the uncertainties of both diagnostic techniques but may also reflect real differences in the plasmas, since the $\mathrm{x}$-ray diagnosed plasmas required an RF power of only $300 \mathrm{~kW}$ (versus $800 \mathrm{~kW}$ ) to drive the same current, other parameters being sensibly the same. 


\section{CONCLUSIONS}

The results from the vertical viewing ECE diagnostic clearly indicate the potential for detailed analysis of the electron velocity distribution. Present limitations include uncertainties in calibration and the atmospheric water vapor line, both of which can be improved upon in principle. The inherent problem of harmonic superposition cannot be alleviated completely, and limits the information available for very energetic distributions.

Distributions measured during lower hybrid current drive and low density ohmic plasmas both show substantial parallel enhancement, with the current drive case having the larger perpendicular spread. Directional temperatures and other parameters have been given to quantify the distributions' parallel and perpendicular characteristics. Semi-quantitative comparisons with theory using these quantities indicate general agreement but a full comparison is difficult owing to the limitations on both the theory and the experiment. Agreement with other diagnostic measurements appears satisfactory.

\section{ACKNOWLEDGEMENTS}

We are grateful for the support of the Alcator $C$ Tokamak experimental staff. This work was funded by the U. S. D.O.E. contract No. DOE-AC02-78ET51013. 


\section{REFERENCES}

${ }^{1}$ H. P. Freund and C.S. Wu, Phys. Fluids 20, 963 (1977).

2.S. Tamor, Science Applications, Inc. LAPS-26 SA177-593-LJ, April (1977).

${ }^{3}$ H.P. Freund, C.S. Wu, L. C. Lee, and D. Dillenburg, Phys. Fluids 21, 1502 (1978)

4.G. Giruzzi, I. Fidone, G. Granata, and R.L. Meyer, Phys. Fluids 27, 1704 (1984).

5. C.M.Celata and D. A. Boyd, Nucl. Fusion 17, 735 (1977).

6.I. Fidone, G. Granata, R.L. Meyer, E.H. Jornada, R.S. Schneider, and L.F. Ziebell, Phys. Fluids 23, 1336 (1980).

7.D. Winske, and D.A. Boyd, Phys. Fluids 26, 755 (1983).

8.D. Winske, Th. Peter, and D.A. Boyd, Phys. Fluids 26, 3497 (1983).

${ }^{9}$ R.F. Ellis, D.B. Robinson, and G.D. Tsakiris, Phys. Rev. A. 26, 3693 (1982).

${ }^{10}$ T. Uckan, and N.A. Uckan, Phys. Fluids 25, 2372 (1982).

${ }^{11}$ A. Murdoch, D.A. Boyd, and R.F. Ellis, Bull. Am. Phys. Soc. Vol. 30, 1420 (1985).

12.F.J. Stauffer, D.A. Boyd, and A.W. Trivelpiece, Physics Letters 57A, 401 (1977).

${ }^{13 . G . D . ~ T s a k i r i s, ~ D . A . ~ B o y d, ~ D . A . ~ H a m m e r, ~ A . W . ~ T r i v e l p i e c e, ~ a n d ~ R . C . ~ D a v i d s o n, ~ P h y s . ~}$ Fluids 21, 2050 (1978).

14.P. Brossier, A.E. Costley, D.S. Komm, G. Ramponi, and S. Tamor, Plasma Phys. and Controlled Nucl. Fusion Research Vol. 1, 401 IAEA (1977).

15.D.J. Campbell, A. Berhagen, and S.E. Kissel, Nucl. Fusion 24, 297 (1984).

${ }^{16}$ H.W. Piekaar, and W.R. Rutgers, Rijnhuizen 80, 128 November (1980).

17.E. Mazzucato, P. Efthimion, and I. Fidone, Nucl. Fusion 25, 1681 (1985).

18.I.H. Hutchinson, and D.S. Komm, Nucl. Fusion 17, 1077 (1977).

19.S. Tamor, Nucl. Fusion 19, 455 (1979).

${ }^{20}$ J.S. Machuzak, private communication, 1986. 
21.D.A. Boyd, Proc. 4th Int. Workshop on ECE and ECRH March 28-30 Rome, 145 (1984).

22.I.H. Hutchinson, and K. Kato, Nucl. Fusion 26, 179 (1986).

23.K.Kato, and I.H. Hutchinson, Rev. Sci. Instrum. 57, 1242 (1986).

24.K.Kato, and I.H. Hutchinson, Rev. Sci. Instrum. 57, 1959 (1986).

${ }^{25}$ K. Kato, M.I.T. Sc.D. Thesis, (1986).

26.W.R. Rutgers, and D.A. Boyd, Phys. Lett. 62A, 498 (1977).

${ }^{27}$ K. Kato, and I.H. Hutchinson, Bull. A.P.S. $\underline{31} 1589$ (1986).

${ }^{28 .}$ K. Kato, and I.H. Hutchinson, Phys. Rev. Lett. 56, 340 (1986).

29.I.H. Hutchinson, K. Kato, and S.C. Texter, Rev. Sci. Instrum. 57, 1951 (1986).

30.P. R. Bevington, Data Reduction and Error Analysis for the Physical Sciences McGrawHill, New York, (1969).

${ }^{31}$ M. Porkolab, B. Lloyd, Y. Takase, P. Bonoli, C. Fiore, R. Gandy, R. Granetz, D. Griffin, D. Gwinn, B. Lipschultz, E. Marmar, S. McCool, A. Pachtman, D. Pappas, R. Parker, P. Pribyl, J. Rice, J.Terry, S. Texter, R. Watterson, and S. Wolfe, Phys. Rev. Lett. 53,1229 (1984).

${ }^{32}$.M. Porkolab, J.J. Schuss, B. Lloyd, Y. Takase, S.Texter, P. Bonoli, C. Fiore, R. Gandy, D. Gwinn, B. Lipschultz, E. Marmar, D. Pappas, R. Parker, and P. Pribyl, Phys. Rev. Lett. 53, 450 (1984).

33.R.J. Bell, Introductory Fourier Transform Spectroscopy, Academic Press, New York, (1969).

34.A.A.M. Oomens, L.Th.M. Ornstein, R.R. Parker, F.C. Schuller, and R.J. Taylor, Phys. Rev. Lett. 36, 255 (1976).

${ }^{35 . H}$. Knoepfel, and D.A. Spong, Nucl. Fusion 19, 785 (1979).

36.V.B. Krapchev, D.W. Hewett, and A. Bers, 4th Int. Symposium on Heating in Toroidal Plasmas, Rome, 674 March (1984).

37.P.T. Bonoli, and R.C. Englade, Phys. Fluids 29, 674 (1986). 
38.V. Fuchs, R.A. Cains, M.M. Shoucri, K. Hizunidis, and A. Bers, Phys. Fluids 28,3619 (1985).

39.V.V. Parail and O.P. Pogutse, Fiz Plasmy 2, 228 (1976).

${ }^{40 .}$ S. von Goeler, et al., Nucl. Fusion 25, 1529 (1985).

${ }^{41}$ S.C. Texter, M.I.T. PFC/RR85-24 (1985). 


\section{Table I}

$$
P_{R F}(\mathrm{~kW})
$$

$I_{p}(\mathrm{kA})$

$$
\bar{n}_{e}\left(\times 10^{20} \mathrm{~m}^{-3}\right)
$$$$
T_{\|}(\mathrm{keV})
$$$$
T_{\perp}(\mathrm{keV})
$$$$
\bar{n}_{t a i l}\left(\times 10^{20} m^{-3}\right)
$$$$
\bar{n}_{t a i l} / \bar{n}_{e}
$$

\section{LHCD}

$$
\left(\Delta \phi=90^{\circ}\right)
$$

Ohmic

800

160

0.4

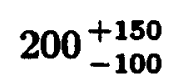

$60_{-15}^{+15}$

$1.2_{-0.4}^{+0.8} \times 10^{-3}$

$3 \times 10^{-3}$

$2 \times 10^{-4}$

\section{LHH}

$$
\Delta \phi=180^{\circ}
$$

400

180

\author{
0.7
}

$$
\begin{array}{ll}
290_{-170}^{+0} & 180 \\
50_{-20}^{+20} & 30_{-8}^{+8}
\end{array}
$$

$6.1_{-2.5}^{+9.5} \times 10^{-4}$

$9.5_{-4.8}^{+4.8} \times 10^{-4}$ 


\section{FIGURE CAPTIONS}

1. Plan and elevation views of the vertical viewing ECE diagnostic system.

2. Extraordinary mode thermal emission measured with and without the dump. ( $\bar{n}_{e} \simeq$ $1.7 \times 10^{20} \mathrm{~m}^{-3}, \mathrm{I}_{\mathrm{p}} \simeq 400 \mathrm{kA}$, and $B_{T}=8 \mathrm{~T}$.)

3. Extraordinary mode nonthermal emission with, (a) $B_{T}=8 \mathrm{~T}, \bar{n}_{e} \sim 1.0 \times 10^{20}$ $\mathrm{m}^{-3}, I_{p} \sim 200 \mathrm{kA}, P_{R F} \sim 500 \mathrm{~kW}$, and without, (b) $B_{T}=8 \mathrm{~T}, \bar{n}_{\mathrm{e}} \sim 0.7 \times 10^{20}$ $\mathrm{m}^{-3}, I_{p} \sim 180 \mathrm{kA}, P_{R F} \sim 400 \mathrm{~kW}$, the viewing dump.

4. Lower hybrid current drive discharge traces. $B_{T}=8 \mathrm{~T}, P_{R F}=800 \mathrm{~kW}$.

5. vertical ECE spectra before and during current drive.

6. Measured and computed spectra for the current drive discharge. $\chi_{\nu}^{2}=0.3$.

7. Distribution function parameters for the current drive discharge. (a) $f_{p}$. (b) $\Lambda$.

8. Distribution function contours for the current drive discharge. Contour magnitudes are given in $\log \left(f_{p}\left(m^{-3}\right)\right)$.

9. Low density ohmic discharge traces. $B_{T}=8 \mathrm{~T}$.

10. Vertical ECE spectra from low density ohmic discharges. (a) O-mode. (b) X-mode.

11. Measured and computed spectra for the low density ohmic discharge. $\chi_{\nu}^{2}=0.2$ from the harmonic ratio. $\chi_{\nu}^{2}=0.6$ from the polarization ratio.

12. Distribution function parameters for the low density ohmic discharge. (a) $f_{p}$. (b) $\Lambda$. ' $\mathrm{P}$ ' and ' $\mathrm{H}$ ' refer to the results of the polarization and the harmonic ratio analyses, respectively.

13. Distribution function contours for the low density ohmic heating discharge. (a) 'Average'. (b) From harmonic ratio. (c) From polarization ratio. Contour magnitudes are given in $\log \left(f_{p}\left(m^{-3}\right)\right)$.

14. $F_{\|}$and $<p_{\perp}^{2}>$ as a function of $p_{\|}$for the lower hybrid current drive discharge distribution.

15. $F_{\|}$and $<p_{\perp}^{2}>$ as a function of $p_{\|}$for the low density ohmic discharge distribution. 
16. $F_{\|}$and $\left\langle p_{\perp}^{2}>\right.$ as a function of $p_{\|}$for the lower hybrid heating discharge distribution. 


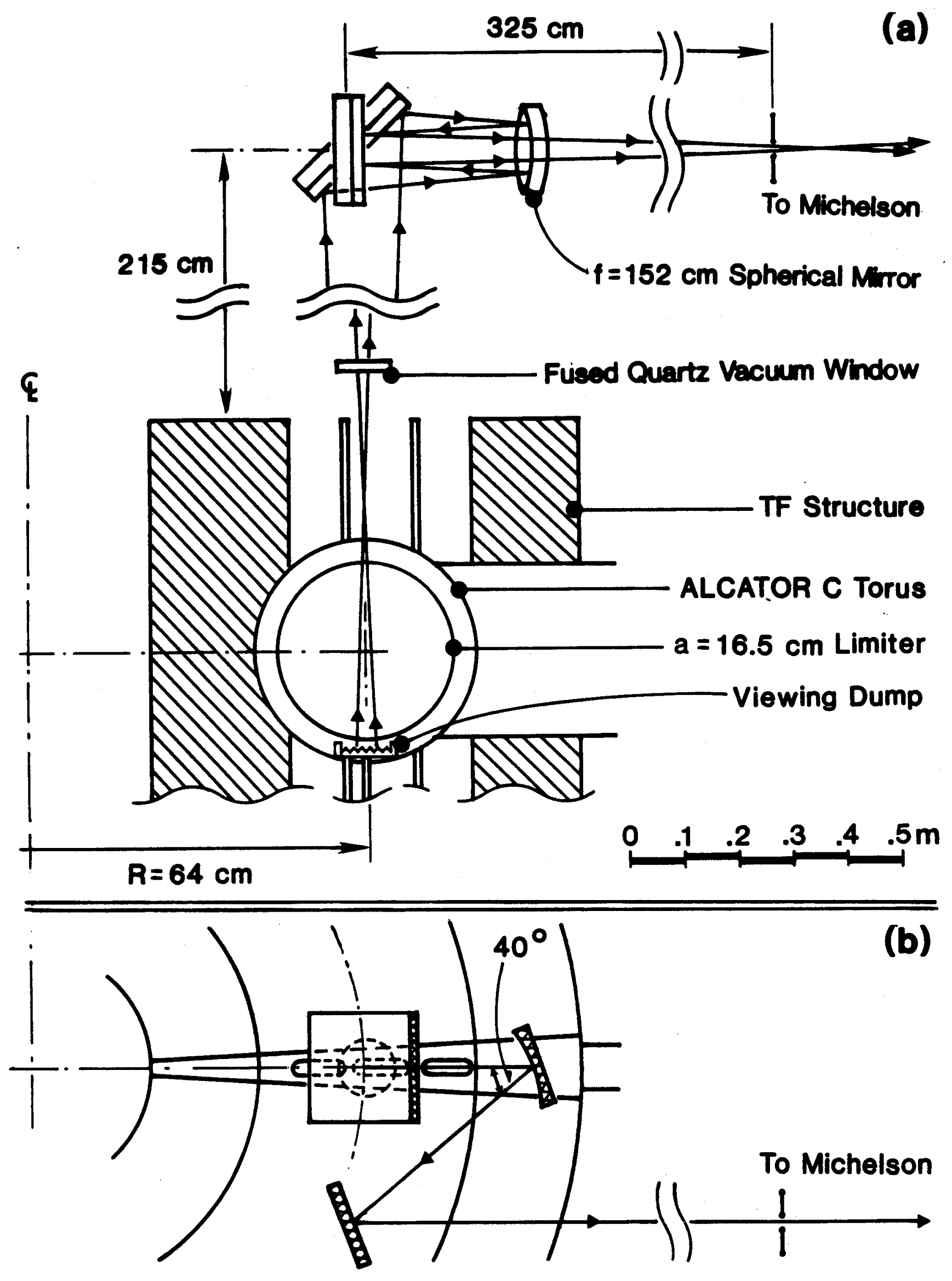

Figure 1 


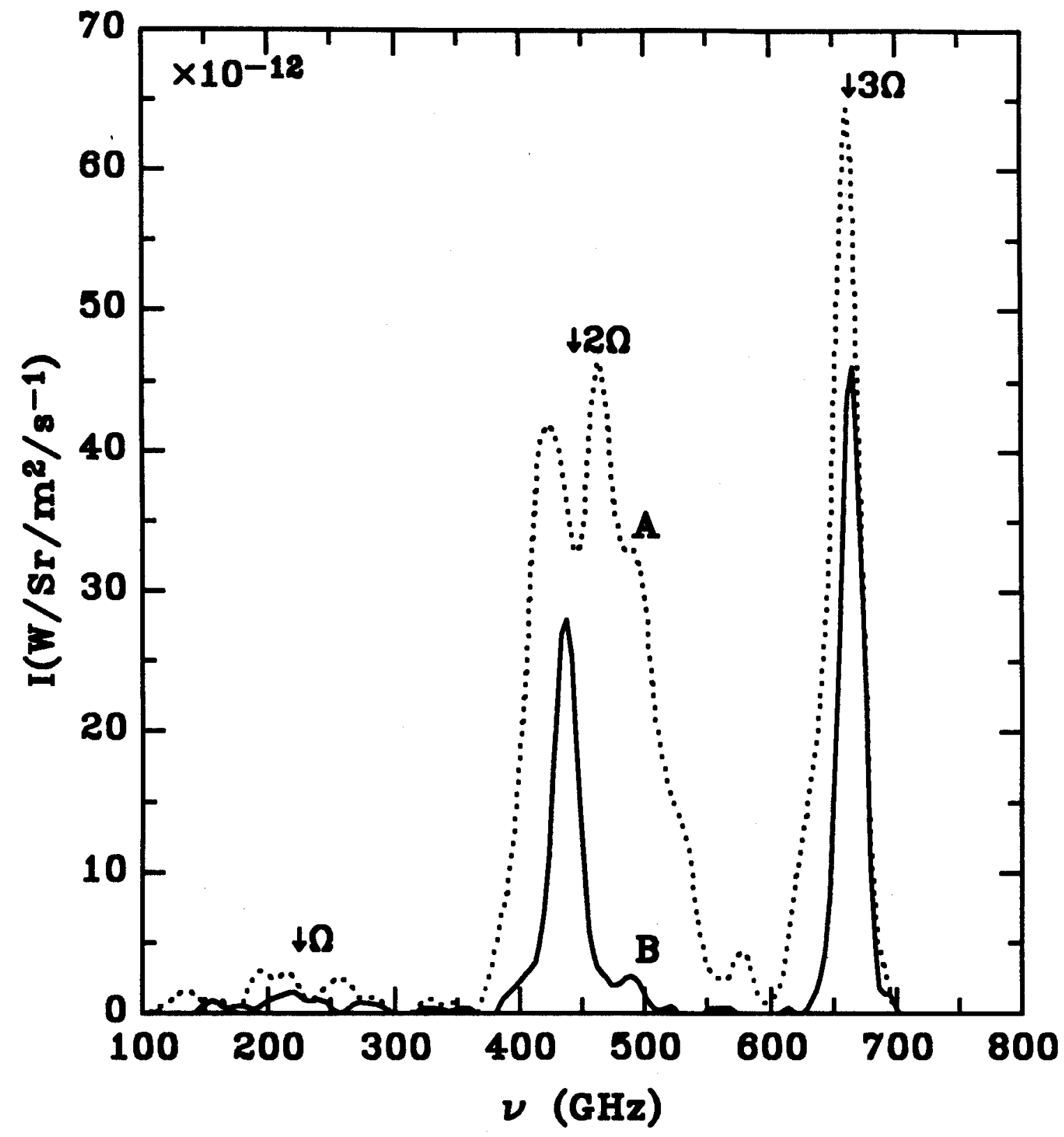

Figure 2 

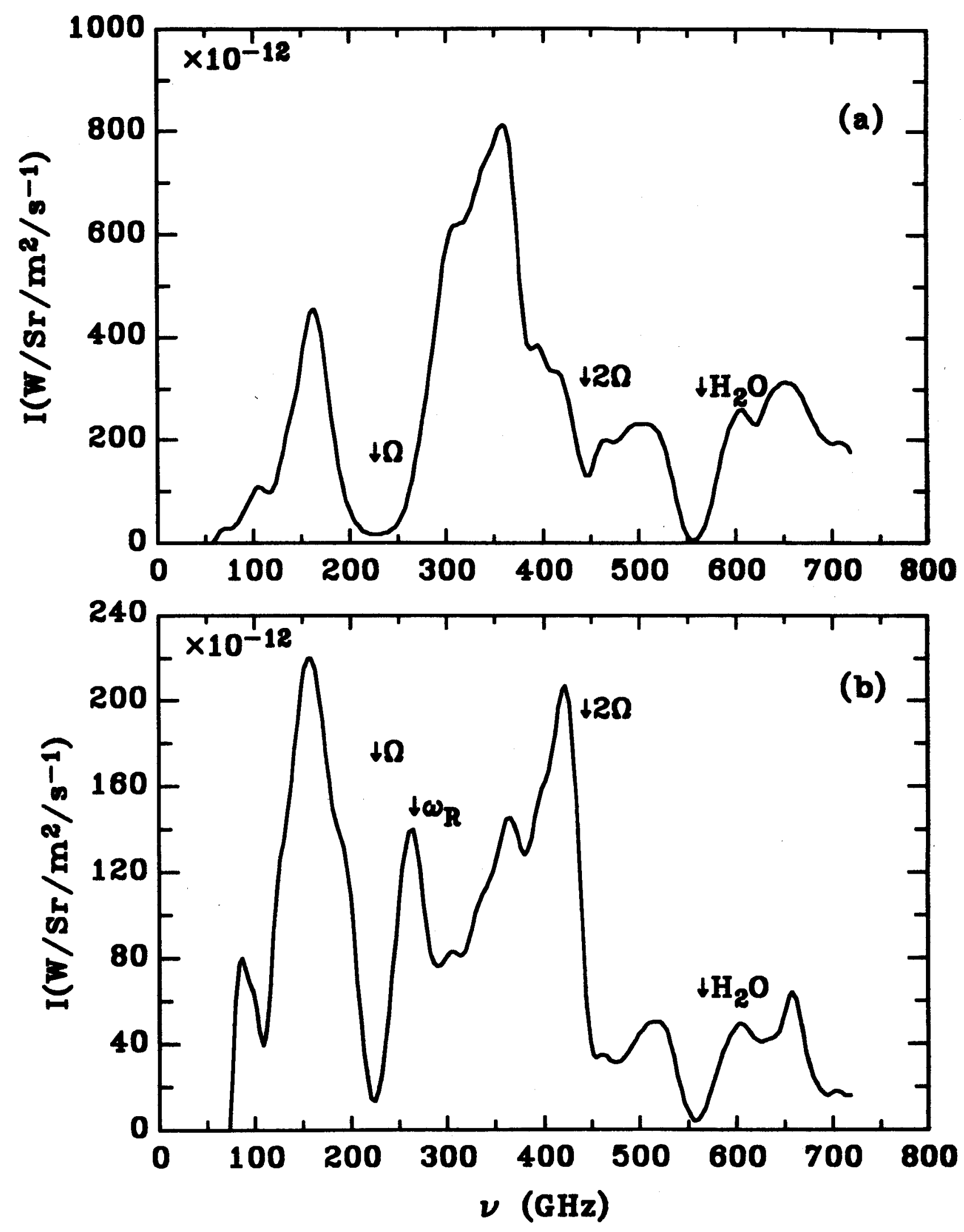

Figure 3 


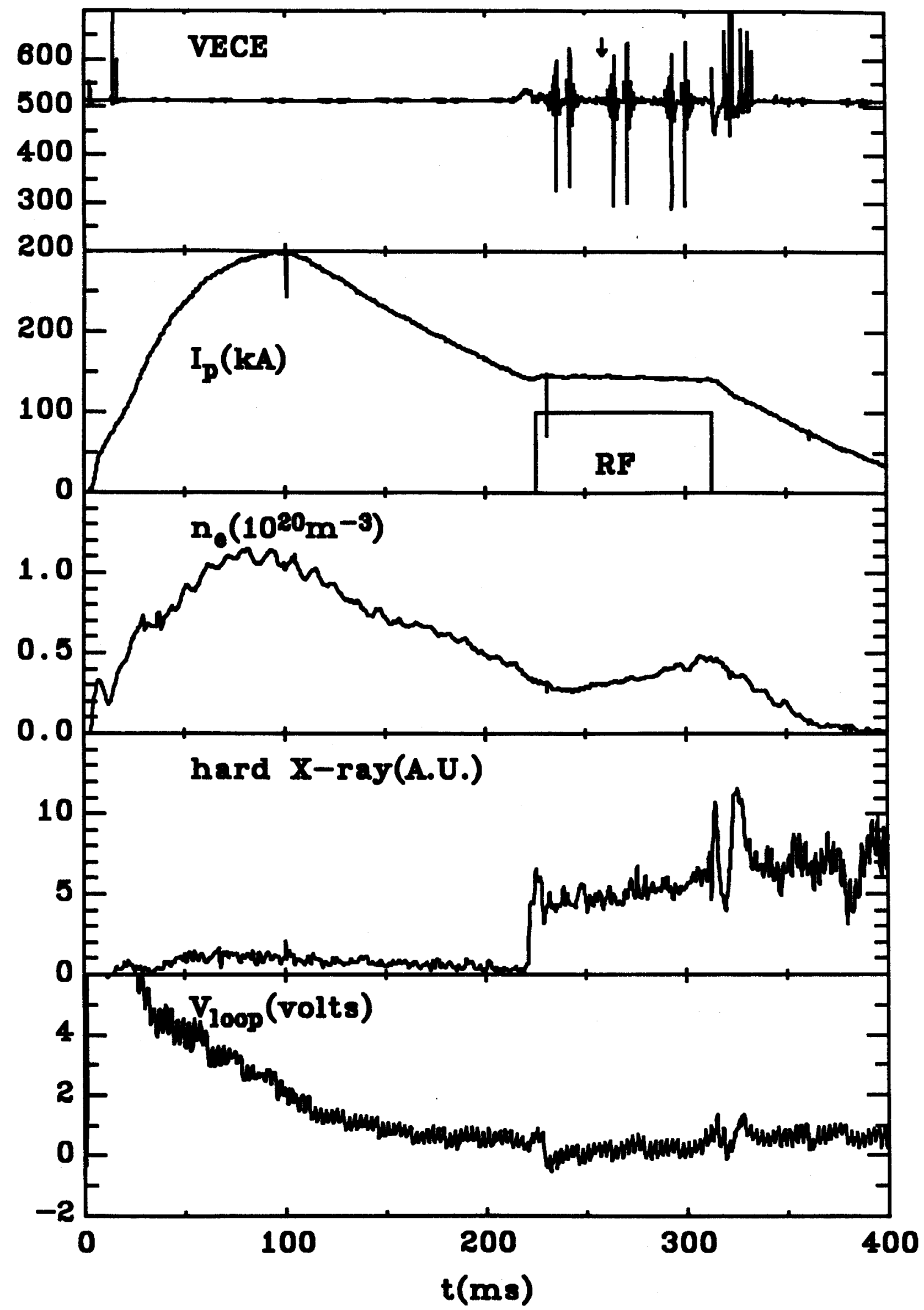

Figure 4 


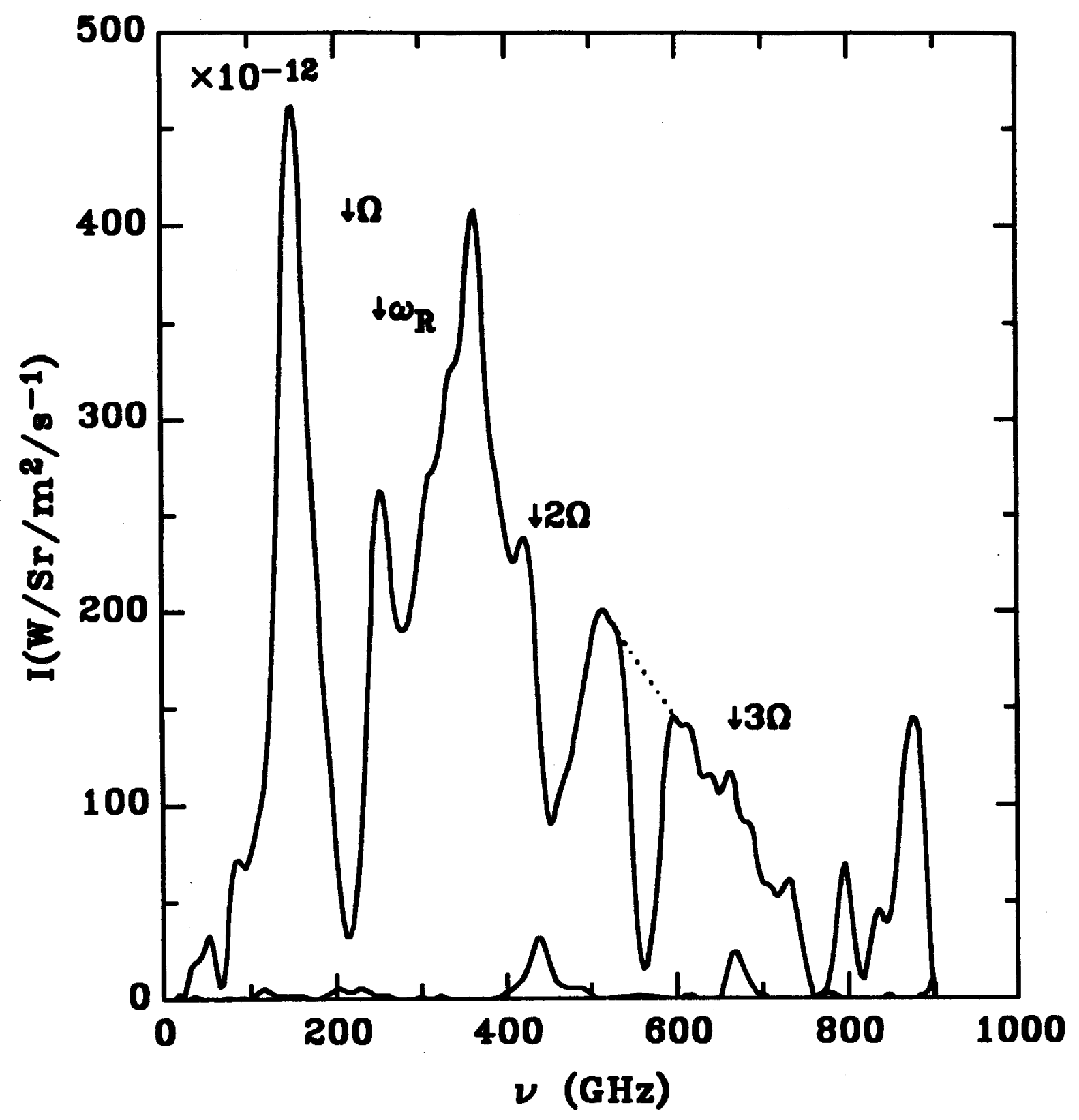

Figure 5 


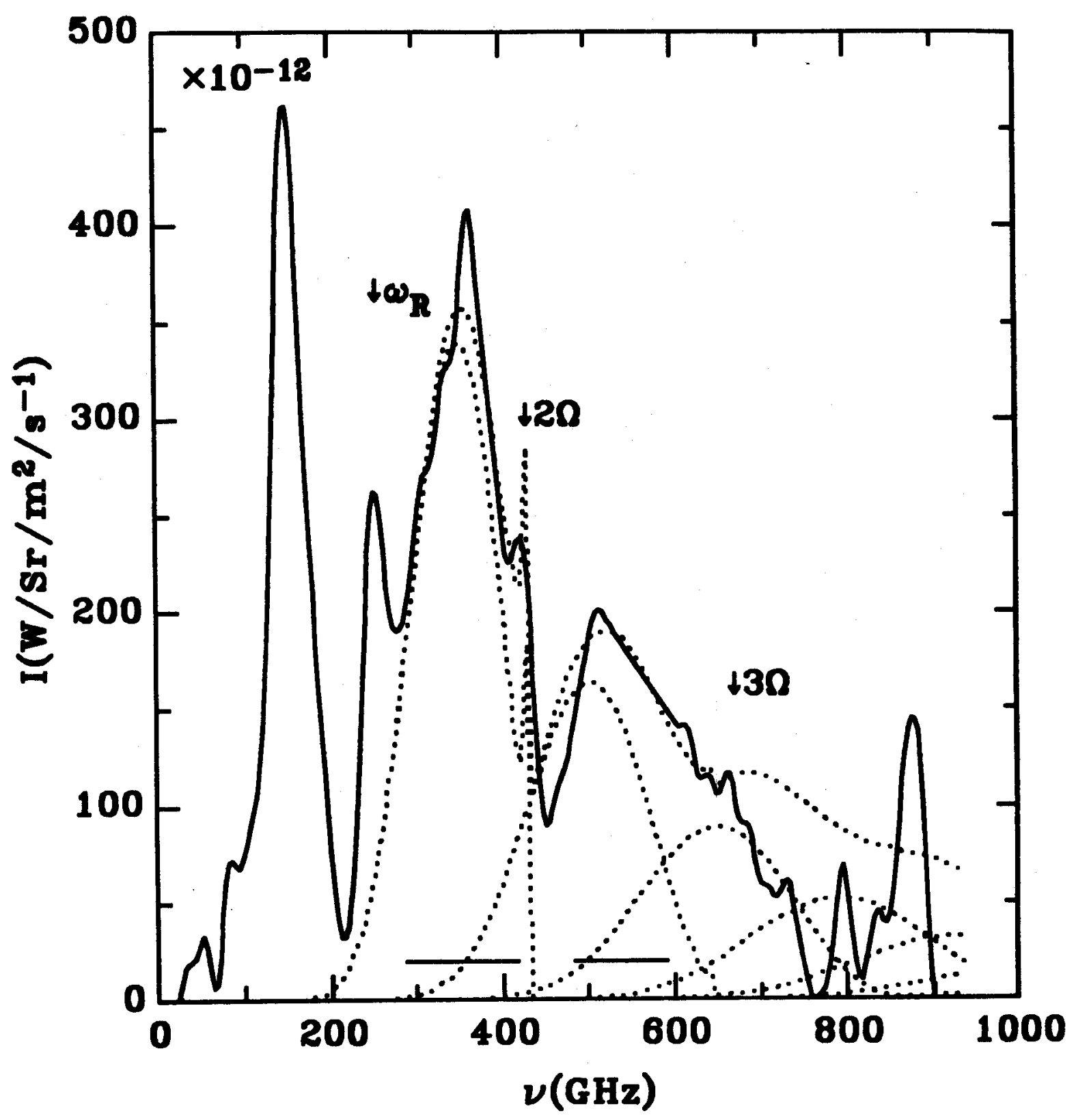

Figure 6 

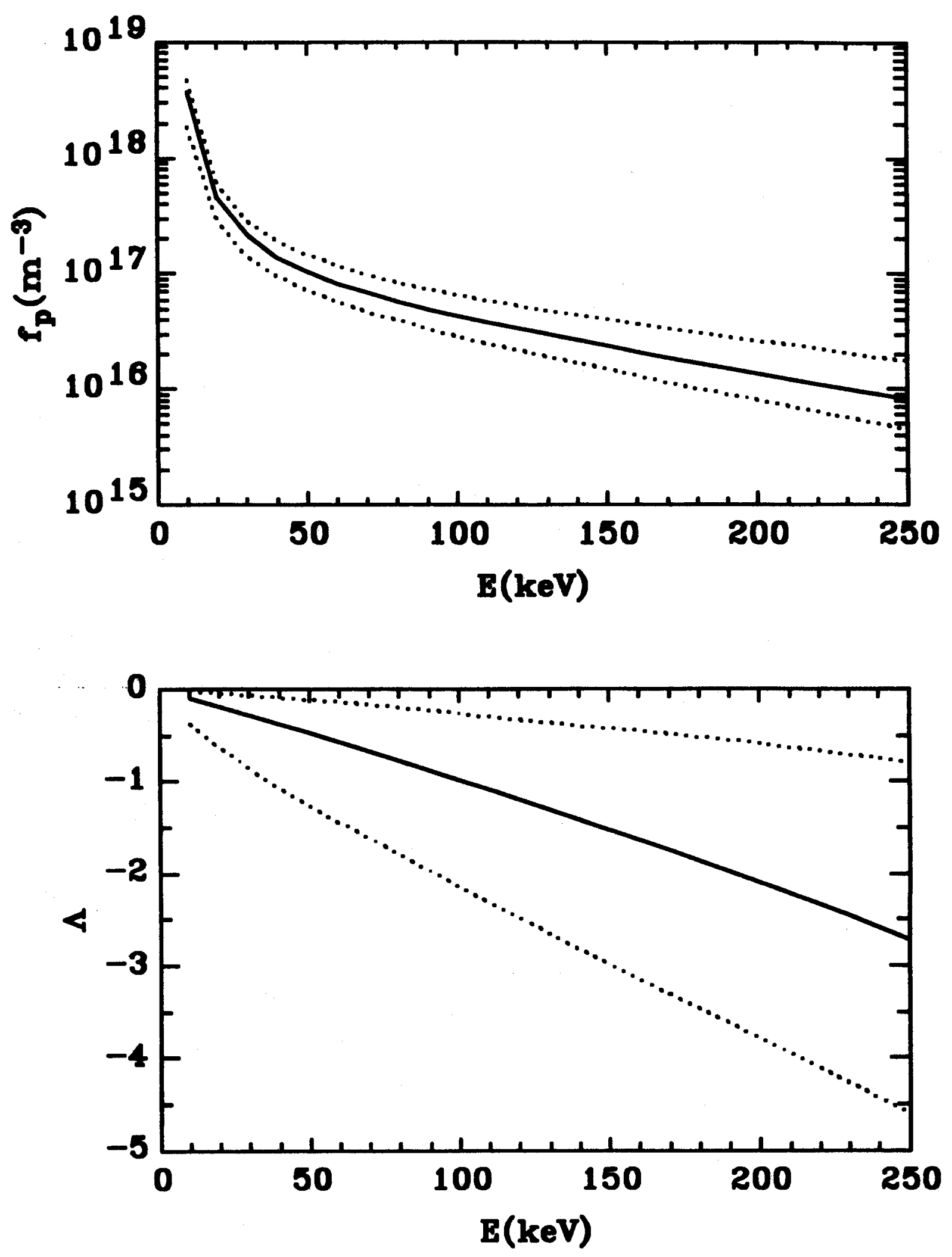

Figure 7 


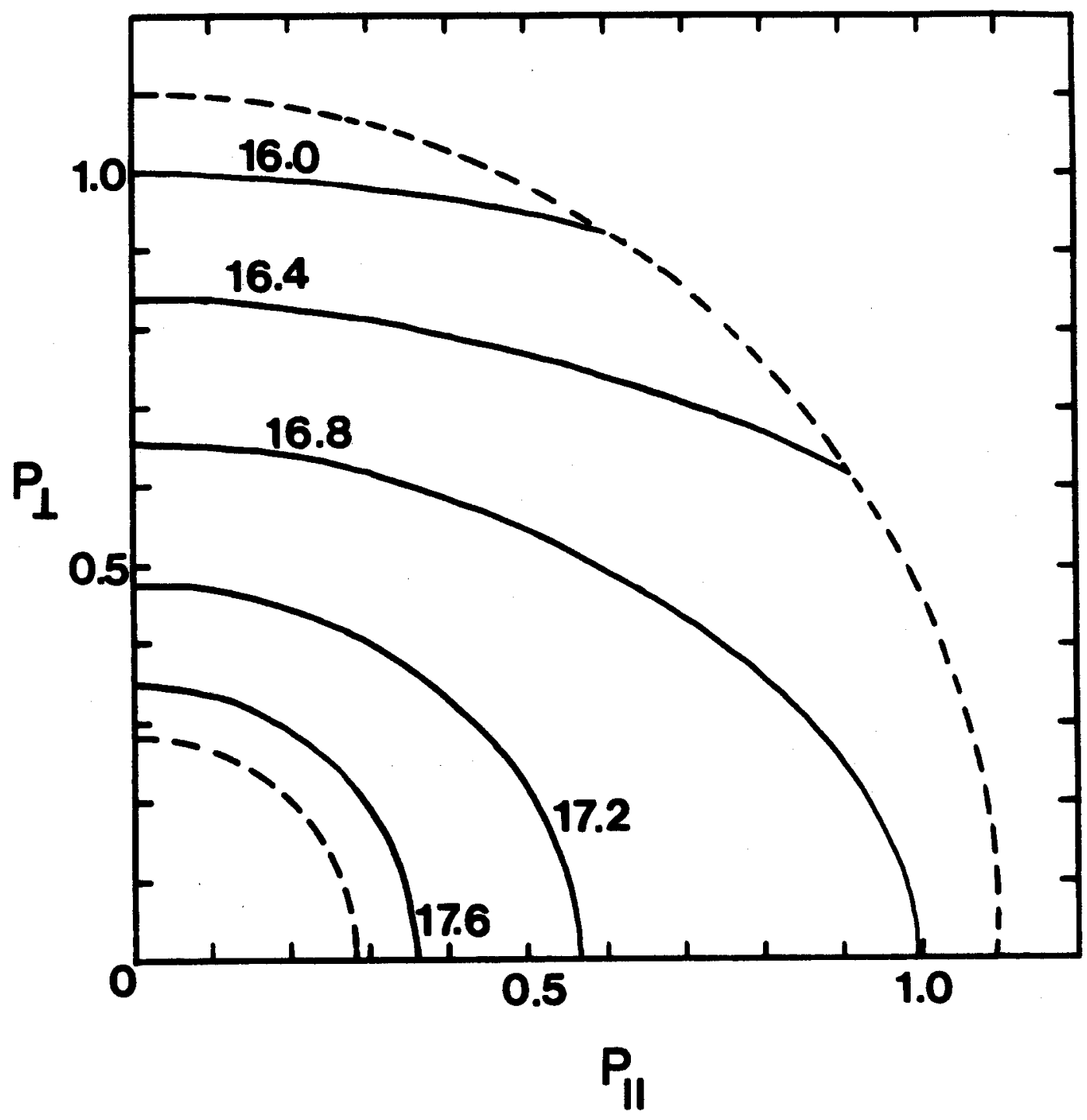

Figure 8 


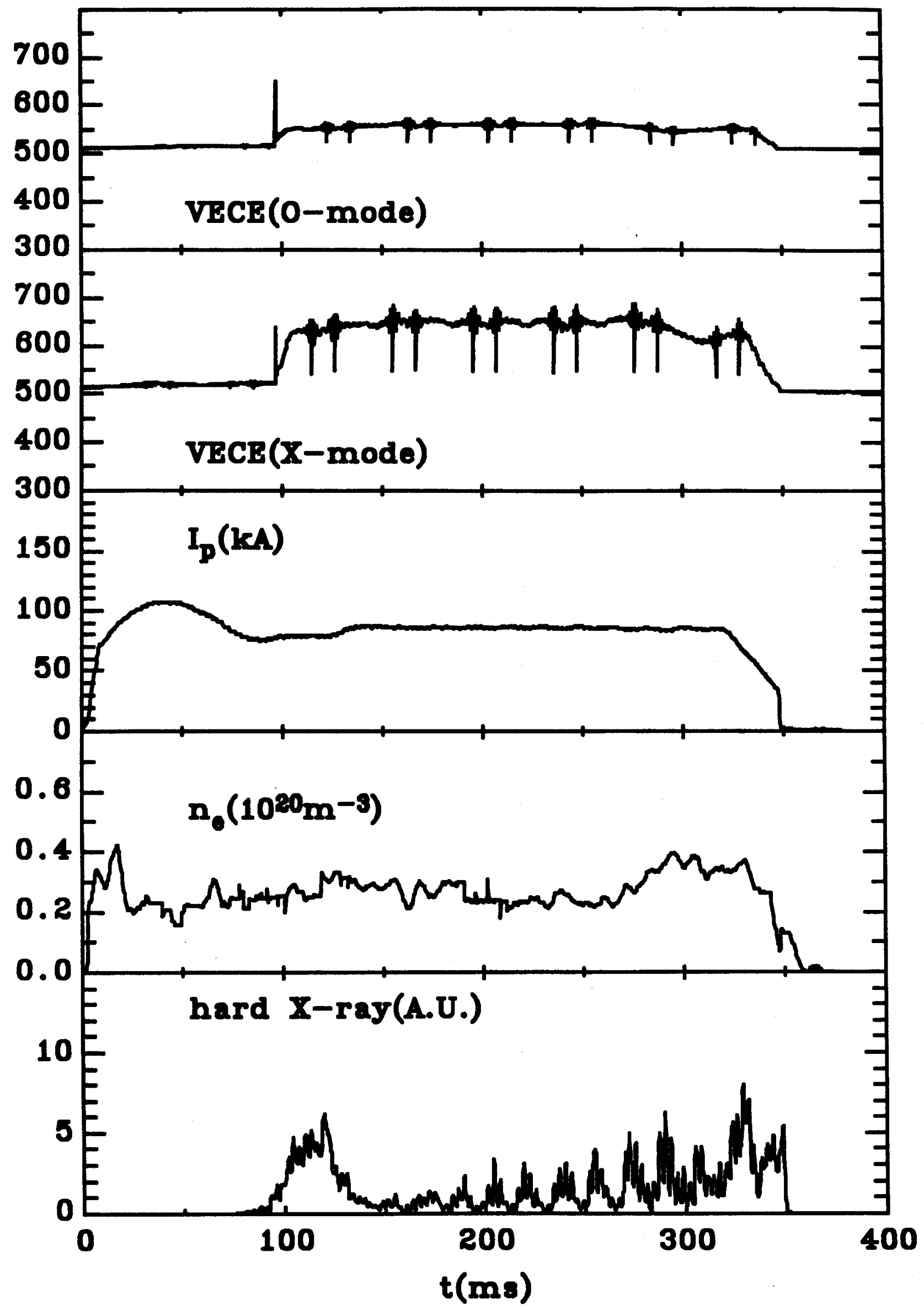

Figure 9 

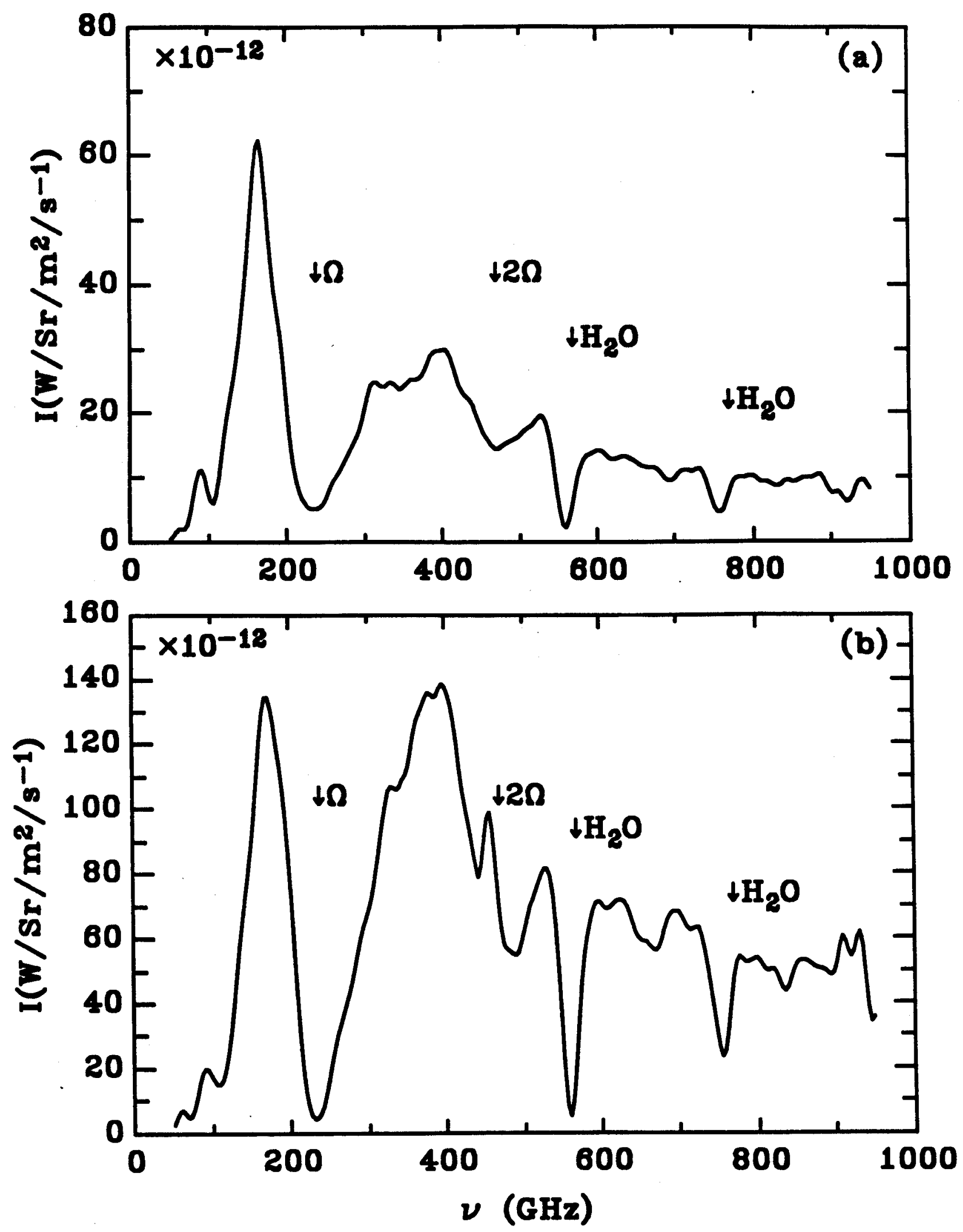

Figure 10 


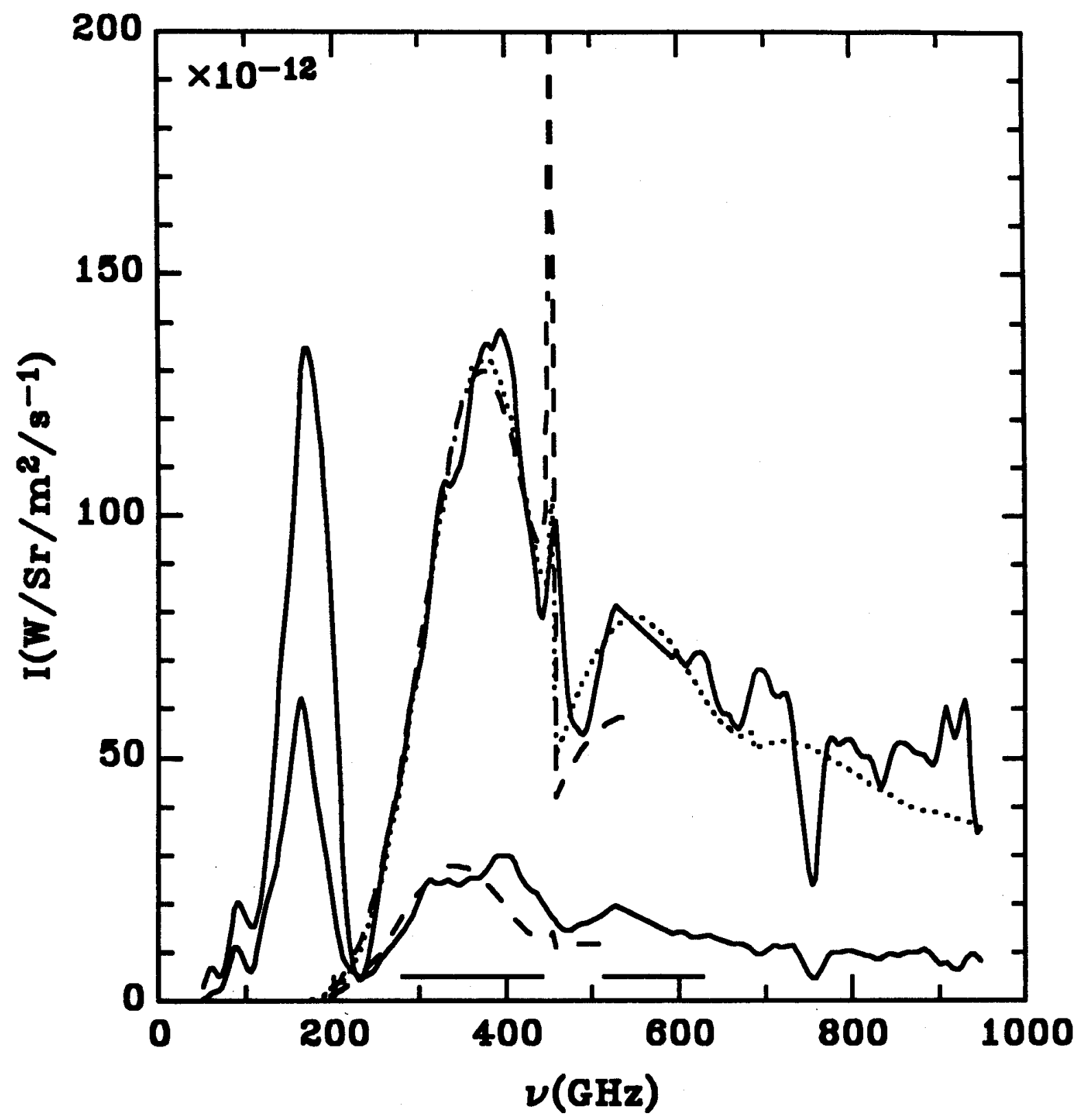

Figure 11 

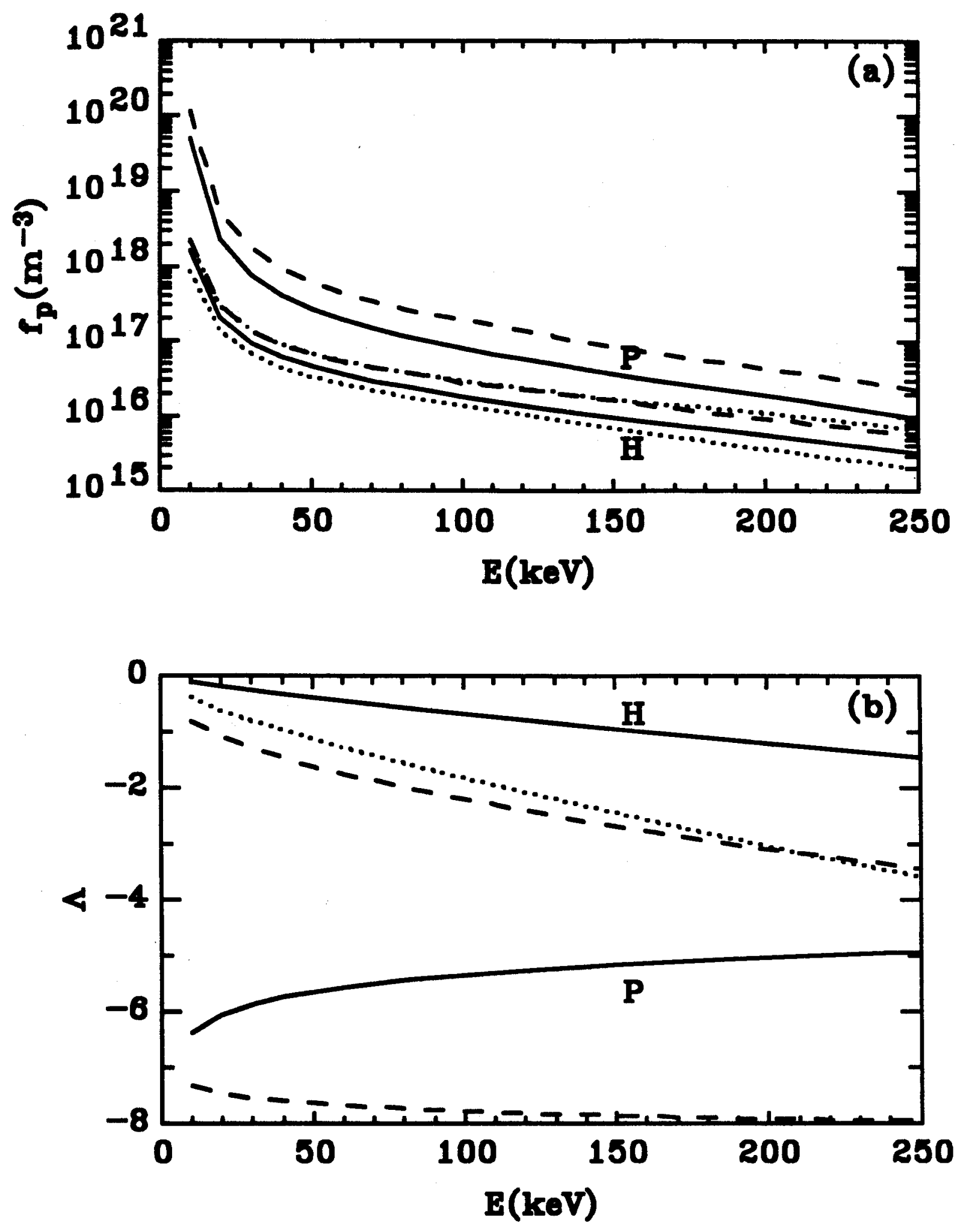

Figure 12 


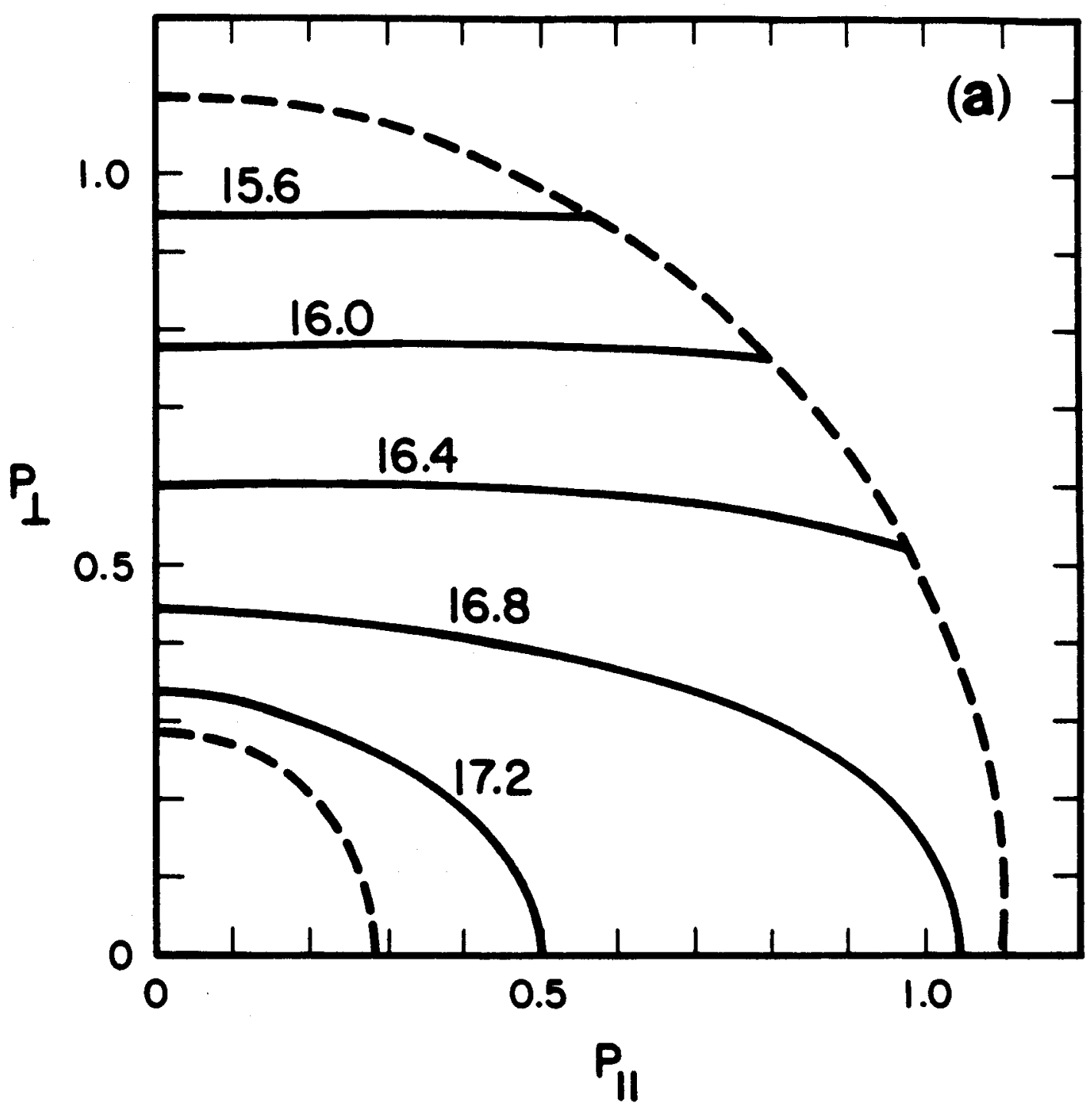

Figure 13 (a)

2076.22 


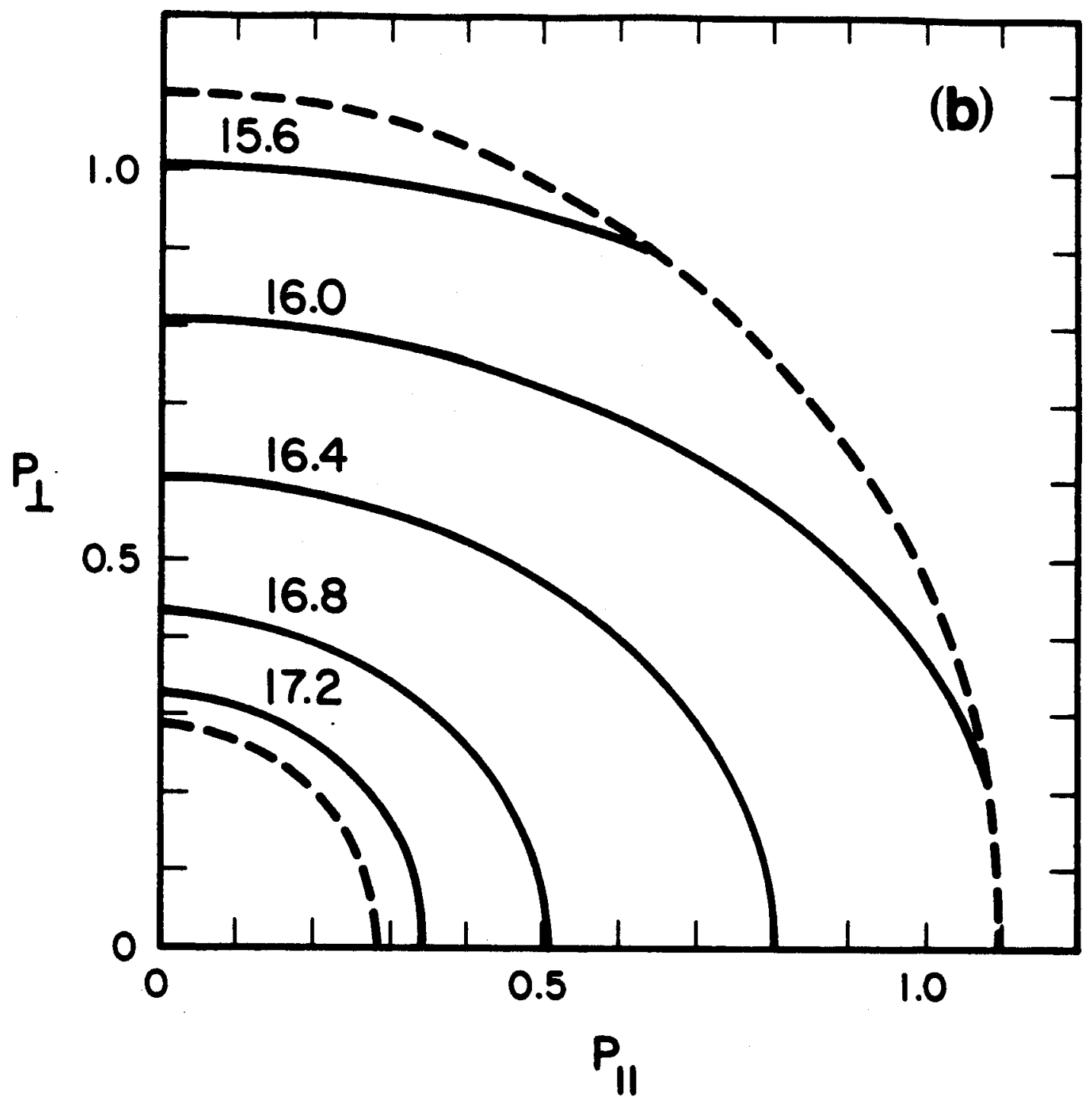

Figure 13 (b) 


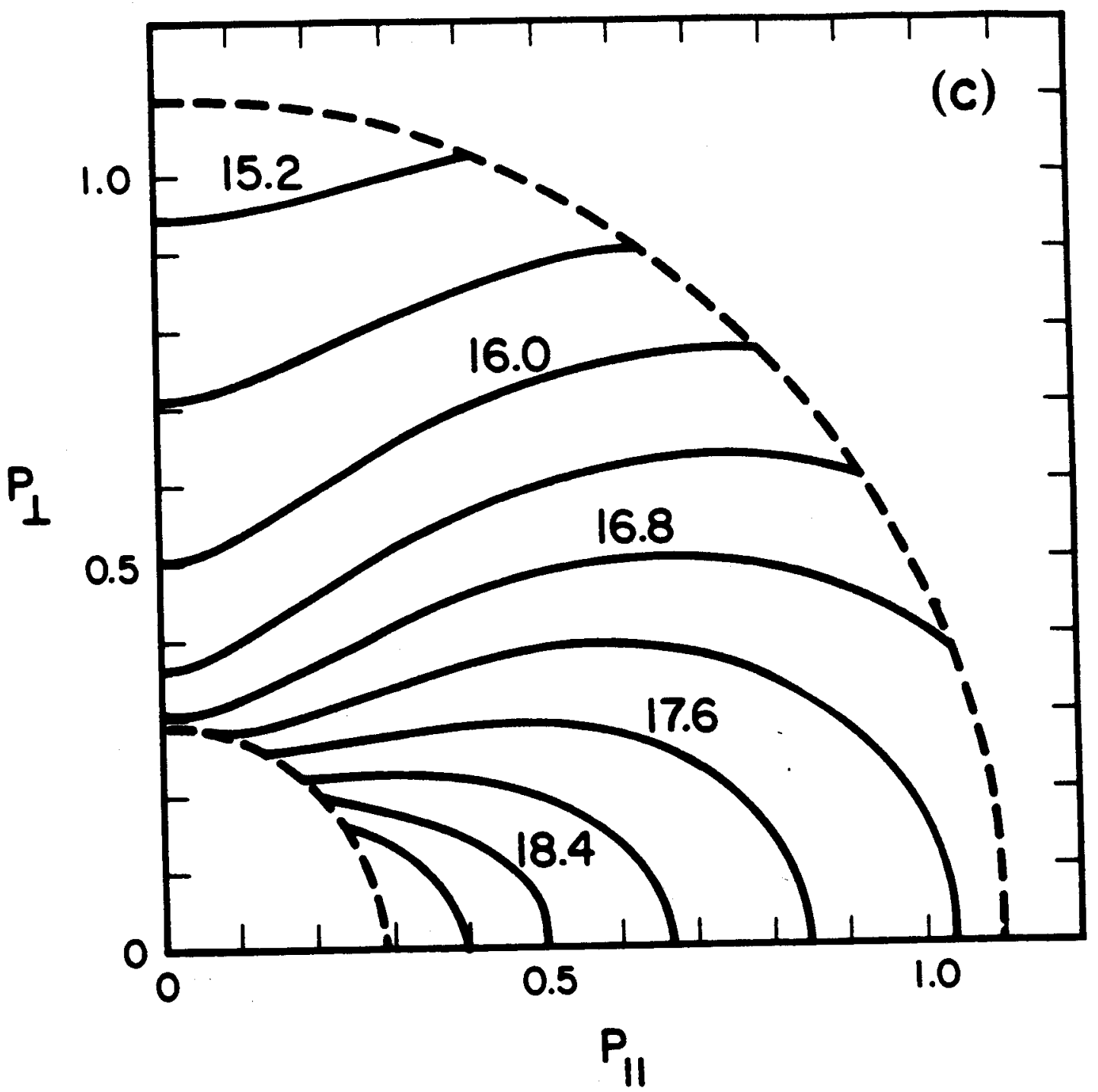

Figure 13 (c)

8896.8 .3 


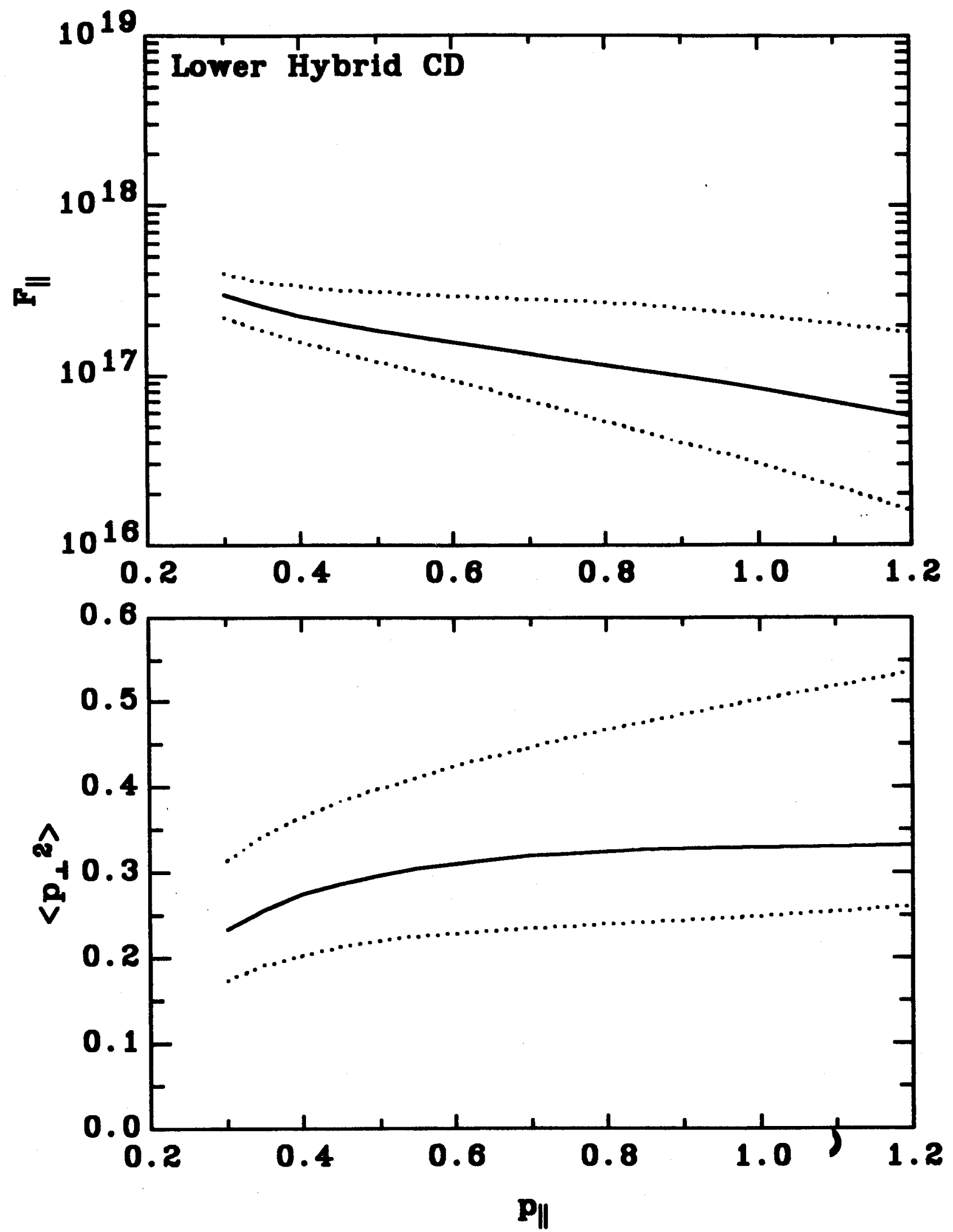

Figure 14 

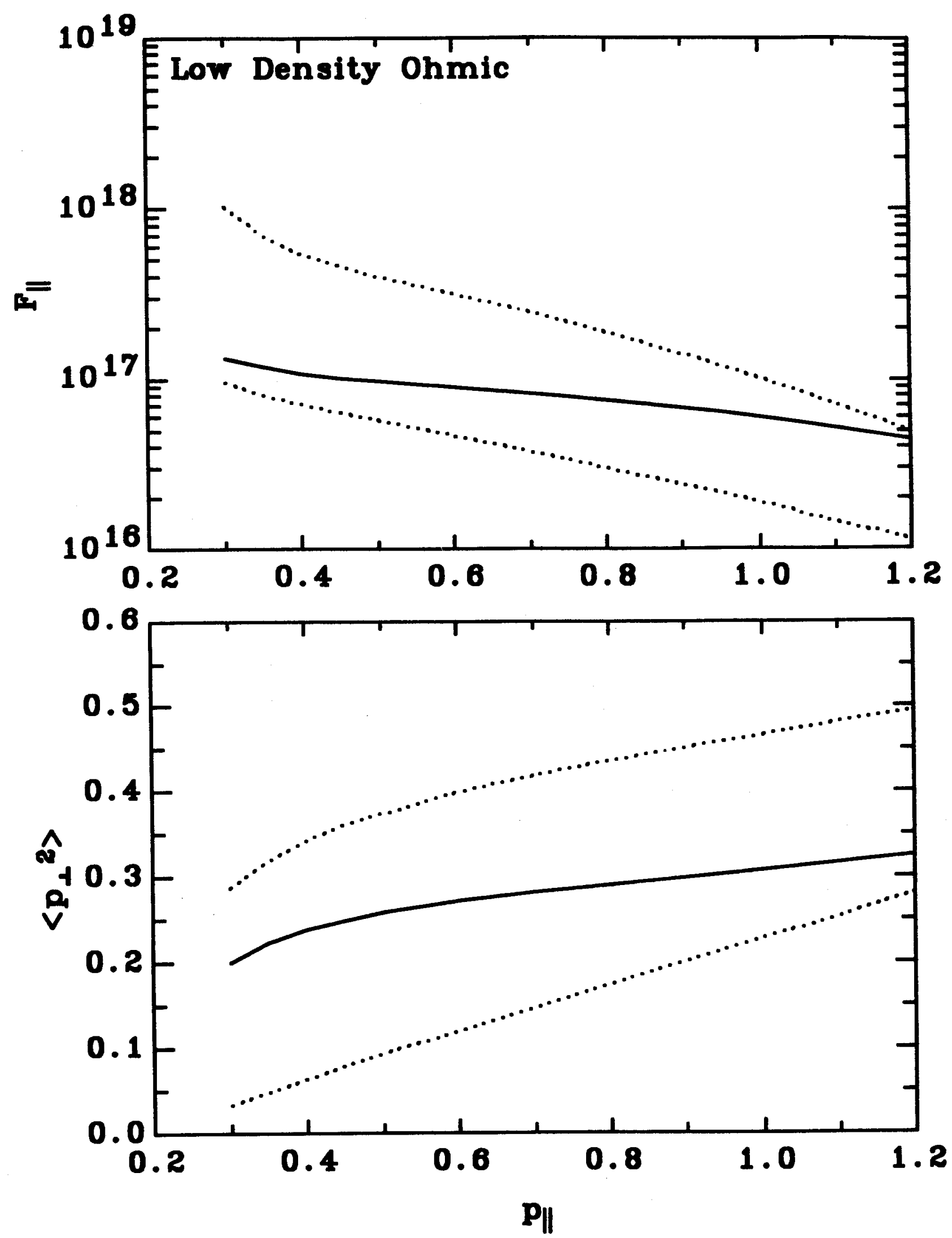

Figure 15 

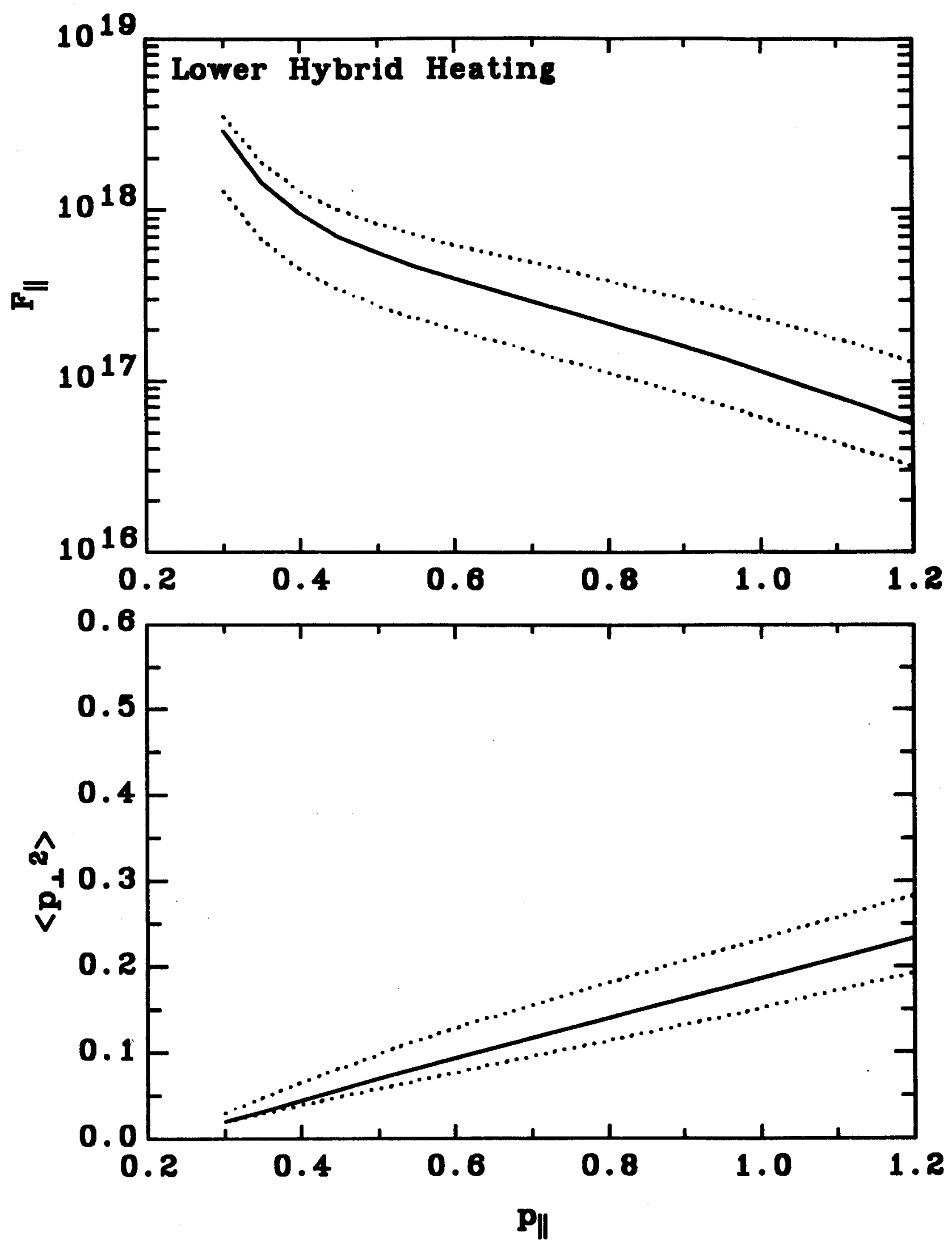

Figure 16 